\title{
Explicit Bounds to Some New Gronwall-Bellman-Type Delay Integral Inequalities in Two Independent Variables on Time Scales
}

\author{
Fanwei Meng, ${ }^{1}$ Qinghua Feng, ${ }^{1,2}$ and Bin Zheng ${ }^{2}$ \\ ${ }^{1}$ School of Mathematical Sciences, Qufu Normal University, Qufu 273165, China \\ ${ }^{2}$ School of Science, Shandong University of Technology, Zibo 255049, China \\ Correspondence should be addressed to Fanwei Meng, fengqinghua1978@126.com
}

Received 20 April 2011; Accepted 8 August 2011

Academic Editor: Bernard Geurts

Copyright (C) 2011 Fanwei Meng et al. This is an open access article distributed under the Creative Commons Attribution License, which permits unrestricted use, distribution, and reproduction in any medium, provided the original work is properly cited.

Some new Gronwall-Bellman-type delay integral inequalities in two independent variables on time scales are established, which provide a handy tool in the research of qualitative and quantitative properties of solutions of delay dynamic equations on time scales. The established inequalities generalize some of the results in the work of Zhang and Meng 2008, Pachpatte 2002, and Ma 2010.

\section{Introduction}

During the past decades, with the development of the theory of differential and integral equations, a lot of integral and difference inequalities have been discovered, which play an important role in the research of boundedness, global existence, stability of solutions of differential and integral equations as well as difference equations. In these established inequalities, Gronwall-Bellman-type inequalities are of particular importance as these inequalities provide explicit bounds for unknown functions, and much effort has been done for developing such inequalities (e.g., see [1-13] and the references therein). On the other hand, Hilger [14] initiated the theory of time scales as a theory capable containing both difference and differential calculus in a consistent way. Since then many authors have expounded on various aspects of the theory of dynamic equations on time scales (e.g., see [15-17] and the references therein). In these investigations, integral inequalities on time scales have been paid much attention by many authors, and a lot of integral inequalities on time scales have been established (e.g., see [18-26]), which have been designed to unify continuous and discrete analysis and play an important role in the research of qualitative and quantitative properties of solutions of certain dynamic equations on time scales. But 
to our knowledge, Gronwall-Bellman-type delay integral inequalities on time scales have been paid little attention in the literature so far. Recent results in this direction include the works of Li [27] and Ma and Pečarić [28] to our best knowledge. Furthermore, nobody has studied Gronwall-Bellman-type delay integral inequalities in two independent variables on time scales.

Our aim in this paper is to establish some new Gronwall-Bellman-type delay integral inequalities in two independent variables on time scales, which unify some known continuous and discrete analysis. New explicit bounds for unknown functions are obtained due to the presented inequalities. We will also present some applications for our results.

First we will give some preliminaries on time scales and some universal symbols for further use.

Throughout the paper, $\mathbb{R}$ denotes the set of real numbers and $\mathbb{R}_{+}=[0, \infty)$, while $\mathbb{Z}$ denotes the set of integers. For two given sets $G, H$, we denote the set of maps from $G$ to $H$ by $(G, H)$.

A time scale is an arbitrary nonempty closed subset of the real numbers. In this paper, $\mathbb{T}$ denotes an arbitrary time scale. On $\mathbb{T}$ we define the forward and backward jump operators $\sigma \in(\mathbb{T}, \mathbb{T})$ and $\rho \in(\mathbb{T}, \mathbb{T})$ by $\sigma(t)=\inf \{s \in \mathbb{T}, s>t\}$ and $\rho(t)=\sup \{s \in \mathbb{T}, s<t\}$.

Definition 1.1. The graininess $\mu \in\left(\mathbb{T}, \mathbb{R}_{+}\right)$is defined by $\mu(t)=\sigma(t)-t$.

Remark 1.2. Obviously, $\mu(t)=0$ if $\mathbb{T}=\mathbb{R}$ while $\mu(t)=1$ if $\mathbb{T}=\mathbb{Z}$.

Definition 1.3. A point $t \in \mathbb{T}$ is said to be left-dense if $\rho(t)=t$ and $t \neq \inf \mathbb{T}$, right-dense if $\sigma(t)=t$ and $t \neq \sup \mathbb{T}$, left-scattered if $\rho(t)<t$, and right-scattered if $\sigma(t)>t$.

Definition 1.4. The set $\mathbb{T}^{\kappa}$ is defined to be $\mathbb{T}$ if $\mathbb{T}$ does not have a left-scattered maximum, otherwise it is $\mathbb{T}$ without the left-scattered maximum.

Definition 1.5. A function $f \in(\mathbb{T}, \mathbb{R})$ is called rd-continuous if it is continuous at right-dense points and if the left-sided limits exist at left-dense points, while $f$ is called regressive if $1+\mu(t) f(t) \neq 0 . C_{\text {rd }}$ denotes the set of rd-continuous functions, while $\Re$ denotes the set of all regressive and rd-continuous functions, and $\mathfrak{R}^{+}=\{f \mid f \in \mathfrak{R}, 1+\mu(t) f(t)>0, \forall t \in \mathbb{T}\}$.

Definition 1.6. For some $t \in \mathbb{T}^{\kappa}$ and a function $f \in(\mathbb{T}, \mathbb{R})$, the delta derivative of $f$ is denoted by $f^{\Delta}(t)$ and satisfies

$$
\left|f(\sigma(t))-f(s)-f^{\Delta}(t)(\sigma(t)-s)\right| \leq \varepsilon|\sigma(t)-s| \text { for } \forall \varepsilon>0,
$$

where $s \in \mathfrak{U}$, and $\mathfrak{U}$ is a neighborhood of $t$ which can depend on $\varepsilon$.

Similarly, for some $y \in \mathbb{T}^{\kappa}$ and a function $f \in(\mathbb{T} \times \mathbb{T}, \mathbb{R})$, the partial delta derivative of $f$ with respect to $y$ is denoted by $(f(x, y))_{y}^{\Delta}$ or $f_{y}^{\Delta}(x, y)$ and satisfies

$$
\left|f(x, \sigma(y))-f(x, s)-f_{y}^{\Delta}(x, y)(\sigma(y)-s)\right| \leq \varepsilon|\sigma(y)-s| \quad \text { for } \forall \varepsilon>0
$$

where $s \in \mathfrak{U}$, and $\mathfrak{U}$ is a neighborhood of $y$ which can depend on $\varepsilon$.

Remark 1.7. If $\mathbb{T}=\mathbb{R}$, then $f^{\Delta}(t)$ becomes the usual derivative $f^{\prime}(t)$, while $f^{\Delta}(t)=f(t+1)-f(t)$ if $\mathbb{T}=\mathbb{Z}$, which represents the forward difference. 
Definition 1.8. For $a, b \in \mathbb{T}$ and a function $f \in(\mathbb{T}, \mathbb{R})$, the Cauchy integral of $f$ is defined by

$$
\int_{a}^{b} f(t) \Delta t=F(b)-F(a)
$$

where $F^{\Delta}(t)=f(t), t \in \mathbb{T}^{\kappa}$.

Similarly, for $a, b \in \mathbb{T}$ and a function $f \in(\mathbb{T} \times \mathbb{T}, \mathbb{R})$, the Cauchy partial integral of $f$ with respect to $y$ is defined by

$$
\int_{a}^{b} f(x, y) \Delta y=F(x, b)-F(x, a)
$$

where $F_{y}^{\Delta}(x, y)=f(x, y), y \in \mathbb{T}^{\kappa}$.

Definition 1.9. The cylinder transformation $\xi_{h}$ is defined by

$$
\xi_{h}(z)= \begin{cases}\frac{\log (1+h z)}{h}, & \text { if } h \neq 0 \quad\left(\text { for } z \neq-\frac{1}{h}\right) \\ z, & \text { if } h=0\end{cases}
$$

where Log is the principal logarithm function.

Definition 1.10. For $p(x, y) \in \mathfrak{R}$ with respect to $y$, the exponential function is defined by

$$
e_{p}(y, s)=\exp \left(\int_{s}^{y} \xi_{\mu(\tau)}(p(x, \tau)) \Delta \tau\right), \quad \text { for } s, y \in \mathbb{T} .
$$

Remark 1.11. If $\mathbb{T}=\mathbb{R}$, then for $y \in \mathbb{R}$ the following formula holds:

$$
e_{p}(y, s)=\exp \left(\int_{s}^{y} p(x, \tau) d \tau\right), \quad \text { for } s \in \mathbb{T}
$$

If $\mathbb{T}=\mathbb{Z}$, then, for $y \in \mathbb{Z}, e_{p}(y, s)=\prod_{\tau=s}^{y-1}[1+p(x, \tau)]$, for $s \in \mathbb{Z}$ and $s<y$. function.

The following two theorems include some known properties on the exponential

Theorem 1.12. If $p(x, y) \in \Re$ with respect to $y$, then the following conclusions hold:

(i) $e_{p}(y, y) \equiv 1$ and $e_{0}(s, y) \equiv 1$,

(ii) $e_{p}(s, \sigma(y))=(1+\mu(y) p(x, y)) e_{p}(s, y)$,

(iii) if $p \in \mathfrak{R}^{+}$with respect to $y$, then $e_{p}(s, y)>0$ for all $s, y \in \mathbb{T}$,

(iv) if $p \in \mathfrak{R}^{+}$with respect to $y$, then $\ominus p \in \mathfrak{R}^{+}$,

(v) $e_{p}(s, y)=1 / e_{p}(y, s)=e_{\ominus p}(y, s)$, where $(\ominus p)(x, y)=-(p(x, y) / 1+\mu(y) p(x, y))$. 
Theorem 1.13. If $p(x, y) \in \mathfrak{R}$ with respect to $y, y_{0} \in \mathbb{T}$ is a fixed number, then the exponential function $e_{p}\left(y, y_{0}\right)$ is the unique solution of the following initial value problem:

$$
\begin{gathered}
z_{y}^{\Delta}(x, y)=p(x, y) z(x, y), \\
z\left(x, y_{0}\right)=1 .
\end{gathered}
$$

Theorems 1.12-1.13 are similar to [24, Theorems 5.1-5.2]. For more details about the calculus of time scales, we advise to refer to [29].

In the rest of this paper, for the convenience of notation, we always assume that $\mathbb{T}_{0}=$ $\left[x_{0}, \infty\right) \cap \mathbb{T}, \widetilde{\mathbb{T}}_{0}=\left[y_{0}, \infty\right) \cap \mathbb{T}$, where $x_{0}, y_{0} \in \mathbb{T}^{\kappa}$, and furthermore assume that $\mathbb{T}_{0} \subseteq \mathbb{T}^{\kappa}, \widetilde{\mathbb{T}}_{0} \subseteq$ $\mathbb{T}^{\kappa}$.

\section{Main Results}

We will give some lemmas for further use.

Lemma 2.1. Suppose that $X \in \mathbb{T}_{0}$ is a fixed number and $u(X, y), b(X, y) \in C_{\mathrm{rd}}, m(X, y) \in \mathfrak{R}_{+}$ with respect to $y, m(X, Y) \geq 0$; then

$$
u(X, y) \leq a(X, y)+b(X, y) \int_{y_{0}}^{y} m(X, t) u(X, t) \Delta t, \quad y \in \widetilde{\mathbb{T}}_{0}
$$

implies

$$
u(X, y) \leq a(X, y)+b(X, y) \int_{y_{0}}^{y} e_{\bar{m}}(y, \sigma(t)) a(X, t) m(X, t) \Delta t, \quad y \in \widetilde{\mathbb{T}}_{0}
$$

where $\bar{m}(X, y)=m(X, y) b(X, y)$, and $e_{\bar{m}}\left(y, y_{0}\right)$ is the unique solution of the following IVP

$$
z_{y}^{\Delta}(X, y)=m(X, y) z(X, y), \quad z\left(X, y_{0}\right)=1
$$

The proof of Lemma 2.1 is similar to that of [24, Theorem 5.6], and we omit it here.

Lemma 2.2. Under the conditions of Lemma 2.1 and furthermore assuming that $a(x, y)$ is nondecreasing in $y$ for every fixed $x, b(x, y) \equiv 1$, then one has

$$
u(X, y) \leq a(X, y) e_{m}\left(y, y_{0}\right)
$$

Proof. Since $a(x, y)$ is nondecreasing in $y$ for every fixed $x$, then from Lemma 2.1 we have

$$
\begin{aligned}
u(X, y) & \leq a(X, y)+\int_{y_{0}}^{y} e_{m}(y, \sigma(t)) a(X, t) m(X, t) \Delta t \\
& \leq a(X, y)\left[1+\int_{y_{0}}^{y} e_{m}(y, \sigma(t)) m(X, t) \Delta t\right] .
\end{aligned}
$$


On the other hand, from [29, Theorems 2.39 and 2.36 (i)] we have $1+\int_{y_{0}}^{y} e_{m}(y, \sigma(t)) m(X, t) \Delta t=$ $e_{m}\left(y, y_{0}\right)$.

Then collecting the above information we can obtain the desired inequality.

Lemma 2.3 (see [30]). Assume that $a \geq 0, p \geq q \geq 0$, and $p \neq 0$, then for any $K>0$

$$
a^{q / p} \leq \frac{q}{p} K^{(q-p) / p} a+\frac{p-q}{p} K^{q / p}
$$

Theorem 2.4. Suppose that $u, f, g, h, a, b \in C_{\mathrm{rd}}\left(\mathbb{T}_{0} \times \widetilde{\mathbb{T}}_{0}, \mathbb{R}_{+}\right)$and $a, b$ are nondecreasing. $p, q, r, m$ are constants, and $p \geq q \geq 0, p \geq r \geq 0, p \geq m \geq 0, p \neq 0 . \tau_{1} \in\left(\mathbb{T}_{0}, \mathbb{T}\right), \tau_{1}(x) \leq x,-\infty<$ $\alpha=\inf \left\{\tau_{1}(x), x \in \mathbb{T}_{0}\right\} \leq x_{0} . \tau_{2} \in\left(\widetilde{\mathbb{T}}_{0}, \mathbb{T}\right), \tau_{2}(y) \leq y,-\infty<\beta=\inf \left\{\tau_{2}(y), y \in \widetilde{\mathbb{T}}_{0}\right\} \leq y_{0}$. $\phi \in C_{\mathrm{rd}}\left(\left(\left[\alpha, x_{0}\right] \times\left[\beta, y_{0}\right]\right) \cap \mathbb{T}^{2}, \mathbb{R}_{+}\right)$. If for $(x, y) \in \mathbb{T}_{0} \times \widetilde{\mathbb{T}}_{0}, u(x, y)$ satisfies the following inequality:

$$
\begin{aligned}
u^{p}(x, y) \leq & a(x, y)+b(x, y) \int_{y_{0}}^{y} \int_{x_{0}}^{x}\left[f(s, t) u^{q}\left(\tau_{1}(s), \tau_{2}(t)\right)+g(s, t) u^{r}(s, t)\right] \Delta s \Delta t \\
& +b(x, y) \int_{y_{0}}^{y} \int_{x_{0}}^{x} \int_{y_{0}}^{t} \int_{x_{0}}^{s} h(\xi, \eta) u^{m}\left(\tau_{1}(\xi), \tau_{2}(\eta)\right) \Delta \xi \Delta \eta \Delta s \Delta t
\end{aligned}
$$

with the initial condition

$$
\begin{gathered}
u(x, y)=\phi(x, y), \quad \text { if } x \in\left[\alpha, x_{0}\right] \bigcap \mathbb{T} \text {, or } y \in\left[\beta, y_{0}\right] \bigcap \mathbb{T}, \\
\phi\left(\tau_{1}(x), \tau_{2}(y)\right) \leq \alpha^{1 / p}(x, y), \quad \text { if } \tau_{1}(x) \leq x_{0} \text { or } \tau_{2}(y) \leq y_{0}, \forall(x, y) \in \mathbb{T}_{0} \times \widetilde{\mathbb{T}}_{0},
\end{gathered}
$$

then

$$
u(x, y) \leq\left[B_{1}(x, y)+b(x, y) \int_{y_{0}}^{y} e_{\bar{B}_{2}}(y, \sigma(t)) B_{2}(x, t) B_{1}(x, t) \Delta t\right]^{1 / p}, \quad(x, y) \in \mathbb{T}_{0} \times \widetilde{\mathbb{T}}_{0},
$$

where

$$
\begin{aligned}
B_{1}(x, y)= & a(x, y)+b(x, y) \int_{y_{0}}^{y} \int_{x_{0}}^{x}\left[f(s, t) \frac{p-q}{p} K^{q / p}+g(s, t) \frac{p-r}{p} K^{r / p}\right] \Delta s \Delta t \\
& +b(x, y) \int_{y_{0}}^{y} \int_{x_{0}}^{x} \int_{y_{0}}^{t} \int_{x_{0}}^{s} h(\xi, \eta) \frac{p-m}{p} K^{m / p} \Delta \xi \Delta \eta \Delta s \Delta t, \quad \forall K>0, \\
B_{2}(x, y)= & \int_{x_{0}}^{x}\left[f(s, y) \frac{q}{p} K^{(q-p) / p}+g(s, y) \frac{r}{p} K^{(r-p) / p}\right. \\
& \left.+\int_{y_{0}}^{y} \int_{x_{0}}^{s} h(\xi, \eta) \frac{m}{p} K^{(m-p) / p} \Delta \xi \Delta \eta\right] \Delta s, \quad \forall K>0, \\
\bar{B}_{2}(x, y)= & b(x, y) B_{2}(x, y) .
\end{aligned}
$$


Proof. Let the right side of (2.7) be $v(x, y)$. Then

$$
u(x, y) \leq v^{1 / p}(x, y), \quad(x, y) \in \mathbb{T}_{0} \times \widetilde{\mathbb{T}}_{0}
$$

If $\tau_{1}(x) \geq x_{0}$ and $\tau_{2}(y) \geq y_{0}$, then $\tau_{1}(x) \in \mathbb{T}_{0}, \tau_{2}(y) \in \widetilde{\mathbb{T}}_{0}$, and since $a, b$ are nondecreasing we have

$$
u\left(\tau_{1}(x), \tau_{2}(y)\right) \leq v^{1 / p}\left(\tau_{1}(x), \tau_{2}(y)\right) \leq v^{1 / p}(x, y)
$$

If $\tau_{1}(x) \leq x_{0}$ or $\tau_{2}(y) \leq y_{0}$, then from (2.8) we have

$$
u\left(\tau_{1}(x), \tau_{2}(y)\right)=\phi\left(\tau_{1}(x), \tau_{2}(y)\right) \leq a^{1 / p}(x, y) \leq v^{1 / p}(x, y)
$$

From (2.14) and (2.15) we have

$$
u\left(\tau_{1}(x), \tau_{2}(y)\right) \leq v^{1 / p}(x, y), \quad(x, y) \in \mathbb{T}_{0} \times \widetilde{\mathbb{T}}_{0}
$$

Fix $X \in \mathbb{T}_{0}$, and let $x \in\left[x_{0}, X\right] \cap \mathbb{T}, y \in \widetilde{\mathbb{T}}_{0}$; then

$$
\begin{aligned}
v(X, y)= & a(X, y)+b(X, y) \int_{y_{0}}^{y} \int_{x_{0}}^{X}\left[f(s, t) u^{q}\left(\tau_{1}(s), \tau_{2}(t)\right)+g(s, t) u^{r}(s, t)\right] \Delta s \Delta t \\
& +b(X, y) \int_{y_{0}}^{y} \int_{x_{0}}^{X} \int_{y_{0}}^{t} \int_{x_{0}}^{s} h(\xi, \eta) u^{m}\left(\tau_{1}(\xi), \tau_{2}(\eta)\right) \Delta \xi \Delta \eta \Delta s \Delta t \\
\leq & a(X, y)+b(X, y) \int_{y_{0}}^{y} \int_{x_{0}}^{X}\left[f(s, t) v^{q / p}(s, t)+g(s, t) v^{r / p}(s, t)\right. \\
& \left.+\int_{y_{0}}^{t} \int_{x_{0}}^{s} h(\xi, \eta) v^{m / p}(\xi, \eta) \Delta \xi \Delta \eta\right] \Delta s \Delta t .
\end{aligned}
$$

From Lemma 2.3, we have

$$
\begin{gathered}
v^{q / p}(x, y) \leq \frac{q}{p} K^{(q-p) / p} v(x, y)+\frac{p-q}{p} K^{q / p}, \\
v^{r / p}(x, y) \leq \frac{r}{p} K^{(r-p) / p} v(x, y)+\frac{p-r}{p} K^{r / p}, \\
v^{m / p}(x, y) \leq \frac{m}{p} K^{(m-p) / p} v(x, y)+\frac{p-m}{p} K^{m / p}, \quad \forall K>0 .
\end{gathered}
$$


So combining (2.17) and (2.18), it follows that

$$
\begin{aligned}
v(X, y) \leq & a(X, y)+b(X, y) \int_{y_{0}}^{y} \int_{x_{0}}^{X} f(s, t)\left(\frac{q}{p} K^{(q-p) / p} v(s, t)+\frac{p-q}{p} K^{q / p}\right) \Delta s \Delta t \\
& +b(X, y) \int_{y_{0}}^{y} \int_{x_{0}}^{X} g(s, t)\left(\frac{r}{p} K^{(r-p) / p} v(s, t)+\frac{p-r}{p} K^{r / p}\right) \Delta s \Delta t \\
& +b(X, y) \int_{y_{0}}^{y} \int_{x_{0}}^{X} \int_{y_{0}}^{t} \int_{x_{0}}^{s} h(\xi, \eta)\left(\frac{m}{p} K^{(m-p) / p} v(\xi, \eta)+\frac{p-m}{p} K^{m / p}\right) \Delta \xi \Delta \eta \Delta s \Delta t \\
\leq & a(X, y)+b(X, y) \int_{y_{0}}^{y} \int_{x_{0}}^{X}\left[f(s, t) \frac{p-q}{p} K^{q / p}+g(s, t) \frac{p-r}{p} K^{r / p}\right. \\
& +b(X, y) \int_{y_{0}}^{y}\left\{\int _ { x _ { 0 } } ^ { X } \left[f(s, t) \frac{q}{p} K^{(q-p) / p}+g(s, t) \frac{r}{p} K^{(r-p) / p}\right.\right. \\
& \left.\left.+\int_{y_{0}}^{t} \int_{x_{0}}^{s} h(\xi, \eta) \frac{m}{p} K^{(m-p) / p} \Delta \xi \Delta \eta\right] \Delta s\right\} v(X, t) \Delta t \\
= & \left.B_{1}(X, y)+b(X, y) \int_{y_{0}}^{y} B_{2}(X, t) v(X, t) \Delta t, \frac{p-m}{p} K^{m / p} \Delta \xi \Delta \eta\right] \Delta s t
\end{aligned}
$$

where $B_{1}(x, y), B_{2}(x, y)$ are defined in (2.10) and (2.11), respectively. Considering $\bar{B}_{2}(X, y)=$ $b(X, y) B_{2}(X, y)$, by application of Lemma 2.1, we have

$$
v(X, y) \leq B_{1}(X, y)+b(X, y) \int_{y_{0}}^{y} e_{\bar{B}_{2}}(y, \sigma(t)) B_{2}(X, t) B_{1}(X, t) \Delta t, \quad y \in \widetilde{\mathbb{T}}_{0} .
$$

Since $X \in \mathbb{T}_{0}$ is arbitrary, then in fact (2.20) holds for all $x \in \mathbb{T}_{0}$, that is,

$$
v(x, y) \leq B_{1}(x, y)+b(x, y) \int_{y_{0}}^{y} e_{\bar{B}_{2}}(y, \sigma(t)) B_{2}(x, t) B_{1}(x, t) \Delta t, \quad(x, y) \in\left(\mathbb{T}_{0} \times \widetilde{\mathbb{T}}_{0}\right) .
$$

Combining (2.13) and (2.21), we obtain

$$
u(x, y) \leq\left[B_{1}(x, y)+b(x, y) \int_{y_{0}}^{y} e_{\bar{B}_{2}}(y, \sigma(t)) B_{2}(x, t) B_{1}(x, t) \Delta t\right]^{1 / p}, \quad(x, y) \in\left(\mathbb{T}_{0} \times \widetilde{\mathbb{T}}_{0}\right),
$$

which is the desired inequality. 
If we apply Lemma 2.2 instead of Lemma 2.1 at the end of the proof of Theorem 2.4, we obtain the following theorem.

Theorem 2.5. Suppose that $u, f, g, h, a, p, q, r, m, \tau_{1}, \tau_{2}, \alpha, \beta, \phi$ are defined as in Theorem 2.4. If that for $(x, y) \in \mathbb{T}_{0} \times \widetilde{\mathbb{T}}_{0}, u(x, y)$ satisfies the following inequality:

$$
\begin{aligned}
u^{p}(x, y) \leq a(x, y)+\int_{y_{0}}^{y} \int_{x_{0}}^{x}[ & f(s, t) u^{q}\left(\tau_{1}(s), \tau_{2}(t)\right)+g(s, t) u^{r}(s, t) \\
& \left.+\int_{y_{0}}^{t} \int_{x_{0}}^{s} h(\xi, \eta) u^{m}\left(\tau_{1}(\xi), \tau_{2}(\eta)\right) \Delta \xi \Delta \eta\right] \Delta s \Delta t
\end{aligned}
$$

with the initial condition (2.8), then

$$
u(x, y) \leq\left[B_{1}(x, y) e_{B_{2}}\left(y, y_{0}\right)\right]^{1 / p}, \quad(x, y) \in \mathbb{T}_{0} \times \widetilde{\mathbb{T}}_{0}
$$

where

$$
\begin{aligned}
& B_{1}(x, y)= a(x, y)+\int_{y_{0}}^{y} \int_{x_{0}}^{x}\left[f(s, t) \frac{p-q}{p} K^{q / p}+g(s, t) \frac{p-r}{p} K^{r / p}\right] \Delta s \Delta t \\
&+\int_{y_{0}}^{y} \int_{x_{0}}^{x} \int_{y_{0}}^{t} \int_{x_{0}}^{s} h(\xi, \eta) \frac{p-m}{p} K^{m / p} \Delta \xi \Delta \eta \Delta s \Delta t, \quad \forall K>0, \\
& B_{2}(x, y)= \int_{x_{0}}^{x}\left[f(s, y) \frac{q}{p} K^{(q-p) / p}+g(s, y) \frac{r}{p} K^{(r-p) / p}+\int_{y_{0}}^{y} \int_{x_{0}}^{s} h(\xi, \eta) \frac{m}{p} K^{(m-p) / p} \Delta \xi \Delta \eta\right] \Delta s, \\
& \forall K>0 .
\end{aligned}
$$

From Theorems 2.4 and 2.5 we can obtain two direct corollaries.

Corollary 2.6. Under the conditions of Theorem 2.4, if, for $(x, y) \in \mathbb{T}_{0} \times \widetilde{\mathbb{T}}_{0}, u(x, y)$ satisfies the following inequality:

$$
\begin{aligned}
u(x, y) \leq & a(x, y)+\int_{y_{0}}^{y} \int_{x_{0}}^{x}\left[f(s, t) u\left(\tau_{1}(s), \tau_{2}(t)\right)+g(s, t) u(s, t)\right] \Delta s \Delta t \\
& +\int_{y_{0}}^{y} \int_{x_{0}}^{x} \int_{y_{0}}^{t} \int_{x_{0}}^{s} h(\xi, \eta) u\left(\tau_{1}(\xi), \tau_{2}(\eta)\right) \Delta \xi \Delta \eta \Delta s \Delta t
\end{aligned}
$$

with the initial condition (2.8) ( $p=1)$, then

$$
u(x, y) \leq a(x, y)+\int_{y_{0}}^{y} e_{B_{2}}(y, \sigma(t)) B_{2}(x, t) a(x, t) \Delta t, \quad(x, y) \in \mathbb{T}_{0} \times \widetilde{\mathbb{T}}_{0},
$$


where

$$
B_{2}(x, y)=\int_{x_{0}}^{x}\left[f(s, y)+g(s, y)+\int_{y_{0}}^{y} \int_{x_{0}}^{s} h(\xi, \eta) \Delta \xi \Delta \eta\right] \Delta s
$$

Corollary 2.7. Under the conditions of Theorem 2.5, if, for $(x, y) \in \mathbb{T}_{0} \times \widetilde{\mathbb{T}}_{0}, u(x, y)$ satisfies the following inequality:

$$
\begin{aligned}
u(x, y) \leq & a(x, y)+\int_{y_{0}}^{y} \int_{x_{0}}^{x}\left[f(s, t) u\left(\tau_{1}(s), \tau_{2}(t)\right)+g(s, t) u(s, t)\right] \Delta s \Delta t \\
& +\int_{y_{0}}^{y} \int_{x_{0}}^{x} \int_{y_{0}}^{t} \int_{x_{0}}^{s} h(\xi, \eta) u\left(\tau_{1}(\xi), \tau_{2}(\eta)\right) \Delta \xi \Delta \eta \Delta s \Delta t
\end{aligned}
$$

with the initial condition (2.8) ( $p=1)$, then

$$
u(x, y) \leq a(x, y) e_{B_{2}}\left(y, y_{0}\right), \quad(x, y) \in \mathbb{T}_{0} \times \widetilde{\mathbb{T}}_{0},
$$

where

$$
B_{2}(x, y)=\int_{x_{0}}^{x}\left[f(s, y)+g(s, y)+\int_{y_{0}}^{y} \int_{x_{0}}^{s} h(\xi, \eta) \Delta \xi \Delta \eta\right] \Delta s .
$$

Theorem 2.8. Suppose that $u \in C_{\mathrm{rd}}\left(\mathbb{T}_{0} \times \widetilde{\mathbb{T}}_{0}, \mathbb{R}_{+}\right), f, g, h, \tau_{1}, \tau_{2}$ are defined as in Theorem 2.4, and $\tau_{1}(x) \geq x_{0}, \tau_{2}(y) \geq y_{0}$. If, for $(x, y) \in \mathbb{T}_{0} \times \widetilde{\mathbb{T}}_{0}, u(x, y)$ satisfies the following inequality:

$$
\begin{aligned}
& u(x, y) \\
& \leq \int_{y_{0}}^{y} \int_{x_{0}}^{x}\left[f(s, t) u\left(\tau_{1}(s), \tau_{2}(t)\right)+g(s, t) u(s, t)+\int_{y_{0}}^{t} \int_{x_{0}}^{s} h(\xi, \eta) u\left(\tau_{1}(\xi), \tau_{2}(\eta)\right) \Delta \xi \Delta \eta\right] \Delta s \Delta t
\end{aligned}
$$

then $u(x, y) \equiv 0$.

The proof of Theorem 2.8 is similar to Theorem 2.4, and we omit it here.

Based on Theorem 2.4, we will establish a class of Volterra-Fredholm-type integral inequality on time scales.

Theorem 2.9. Suppose that $u, f_{i}, g_{i}, h_{i} \in C_{\mathrm{rd}}\left(\mathbb{T}_{0} \times \widetilde{\mathbb{T}}_{0}, \mathbb{R}_{+}\right), i=1,2 . a, p, q, r, m, \phi, \tau_{1}, \tau_{2}, \alpha, \beta$ are the same as in Theorem 2.4, and $M \in \mathbb{T}_{0}, N \in \widetilde{\mathbb{T}}_{0}$ are two fixed numbers. If, for $(x, y) \in$ $\left(\left[x_{0}, M\right] \cap \mathbb{T}\right) \times\left(\left[y_{0}, N\right] \cap \mathbb{T}\right), u(x, y)$ satisfies the following inequality:

$$
\begin{aligned}
u^{p}(x, y) \leq & a(x, y)+\int_{y_{0}}^{y} \int_{x_{0}}^{x}\left[f_{1}(s, t) u^{q}\left(\tau_{1}(s), \tau_{2}(t)\right)+g_{1}(s, t) u^{r}\left(\tau_{1}(s), \tau_{2}(t)\right)\right] \Delta s \Delta t \\
& +\int_{y_{0}}^{y} \int_{x_{0}}^{x} \int_{y_{0}}^{t} \int_{x_{0}}^{s} h_{1}(\xi, \eta) u^{m}\left(\tau_{1}(\xi), \tau_{2}(\eta)\right) \Delta \xi \Delta \eta \Delta s \Delta t
\end{aligned}
$$




$$
\begin{aligned}
& +\int_{y_{0}}^{N} \int_{x_{0}}^{M}\left[f_{2}(s, t) u^{q}\left(\tau_{1}(s), \tau_{2}(t)\right)+g_{2}(s, t) u^{r}\left(\tau_{1}(s), \tau_{2}(t)\right)\right] \Delta s \Delta t \\
& +\int_{y_{0}}^{N} \int_{x_{0}}^{M} \int_{y_{0}}^{t} \int_{x_{0}}^{s} h_{2}(\xi, \eta) u^{m}\left(\tau_{1}(\xi), \tau_{2}(\eta)\right) \Delta \xi \Delta \eta \Delta s \Delta t
\end{aligned}
$$

with the initial condition (2.8), then one has

$$
\begin{aligned}
& u(x, y) \\
& \quad \leq\left\{\left[\frac{\lambda+\widetilde{B}_{6}}{1-\widetilde{B}_{5}}\right] \widetilde{B}_{3}(x, y)+\widetilde{B}_{4}(x, y)\right\}^{1 / p}, \quad(x, y) \in\left(\left[x_{0}, M\right] \bigcap \mathbb{T}\right) \times\left(\left[y_{0}, N\right] \bigcap \mathbb{T}\right),
\end{aligned}
$$

provided that $\widetilde{B}_{5}<1$, where

$$
\begin{aligned}
& \lambda=\int_{y_{0}}^{N} \int_{x_{0}}^{M}\left[f_{2}(s, t) \frac{p-q}{p} K^{q / p}+g_{2}(s, t) \frac{p-r}{p} K^{r / p}\right. \\
& \left.+\int_{y_{0}}^{t} \int_{x_{0}}^{s} h_{2}(\xi, \eta) \frac{p-m}{p} K^{m / p} \Delta \xi \Delta \eta\right] \Delta s \Delta t \\
& \tilde{B}_{1}(x, y)=a(x, y)+\int_{y_{0}}^{y} \int_{x_{0}}^{x}\left[f_{1}(s, t) \frac{p-q}{p} K^{q / p}+g_{1}(s, t) \frac{p-r}{p} K^{r / p}\right] \Delta s \Delta t \\
& +\int_{y_{0}}^{y} \int_{x_{0}}^{x} \int_{y_{0}}^{t} \int_{x_{0}}^{s} h_{1}(\xi, \eta) \frac{p-m}{p} K^{m / p} \Delta \xi \Delta \eta \Delta s \Delta t, \quad \forall K>0, \\
& \widetilde{B}_{2}(x, y)=\int_{x_{0}}^{x}\left[f_{1}(s, y) \frac{q}{p} K^{(q-p) / p}+g_{1}(s, y) \frac{r}{p} K^{(r-p) / p}\right. \\
& \left.+\int_{y_{0}}^{y} \int_{x_{0}}^{s} h_{1}(\xi, \eta) \frac{m}{p} K^{(m-p) / p} \Delta \xi \Delta \eta\right] \Delta s, \quad \forall K>0, \\
& \widetilde{B}_{3}(x, y)=1+\int_{y_{0}}^{y} e_{\widetilde{B}_{2}}(y, \sigma(t)) \widetilde{B}_{2}(x, t) \Delta t, \\
& \widetilde{B}_{4}(x, y)=\widetilde{B}_{1}(x, y)+\int_{y_{0}}^{y} e_{\widetilde{B}_{2}}(y, \sigma(t)) \widetilde{B}_{2}(x, t) \widetilde{B}_{1}(x, t) \Delta t, \\
& \widetilde{B}_{5}=\int_{y_{0}}^{N} \int_{x_{0}}^{M}\left[f_{2}(s, t) \frac{q}{p} K^{(q-p) / p} \widetilde{B}_{3}(s, t)+g_{2}(s, t) \frac{r}{p} K^{(r-p) / p} \widetilde{B}_{3}(s, t)\right] \Delta s \Delta t \\
& +\int_{y_{0}}^{N} \int_{x_{0}}^{M} \int_{y_{0}}^{t} \int_{x_{0}}^{s} h_{2}(\xi, \eta) \frac{m}{p} K^{(m-p) / p} \widetilde{B}_{3}(\xi, \eta) \Delta \xi \Delta \eta \Delta s \Delta t
\end{aligned}
$$


Journal of Applied Mathematics

$$
\begin{aligned}
\widetilde{B}_{6}= & \int_{y_{0}}^{N} \int_{x_{0}}^{M}\left[f_{2}(s, t) \frac{q}{p} K^{(q-p) / p} \widetilde{B}_{4}(s, t)+g_{2}(s, t) \frac{r}{p} K^{(r-p) / p} \widetilde{B}_{4}(s, t)\right] \Delta s \Delta t \\
& +\int_{y_{0}}^{N} \int_{x_{0}}^{M} \int_{y_{0}}^{t} \int_{x_{0}}^{s} h_{2}(\xi, \eta) \frac{m}{p} K^{(m-p) / p} \widetilde{B}_{4}(\xi, \eta) \Delta \xi \Delta \eta \Delta s \Delta t .
\end{aligned}
$$

Proof. Let the right side of $(2.33)$ be $v(x, y)$ and

$$
\begin{aligned}
\mu= & \int_{y_{0}}^{N} \int_{x_{0}}^{M}\left[f_{2}(s, t) u^{q}\left(\tau_{1}(s), \tau_{2}(t)\right)+g_{2}(s, t) u^{r}\left(\tau_{1}(s), \tau_{2}(t)\right)\right] \Delta s \Delta t \\
& +\int_{y_{0}}^{N} \int_{x_{0}}^{M} \int_{y_{0}}^{t} \int_{x_{0}}^{s} h_{2}(\xi, \eta) u^{m}\left(\tau_{1}(\xi), \tau_{2}(\eta)\right) \Delta \xi \Delta \eta \Delta s \Delta t .
\end{aligned}
$$

Then

$$
u(x, y) \leq v^{1 / p}(x, y), \quad(x, y) \in\left(\left[x_{0}, M\right] \bigcap \mathbb{T}\right) \times\left(\left[y_{0}, N\right] \bigcap \mathbb{T}\right)
$$

Similar to the process of (2.14)-(2.16) one has

$$
u\left(\tau_{1}(x), \tau_{2}(y)\right) \leq v^{1 / p}(x, y), \quad(x, y) \in\left(\left[x_{0}, M\right] \bigcap \mathbb{T}\right) \times\left(\left[y_{0}, N\right] \bigcap \mathbb{T}\right)
$$

Fix $X \in\left[x_{0}, M\right] \cap \mathbb{T}$, and let $x \in\left[x_{0}, X\right] \cap \mathbb{T}, y \in\left[y_{0}, N\right] \cap \mathbb{T}$. Then

$$
\begin{aligned}
v(X, y)= & a(X, y)+\mu+\int_{y_{0}}^{y} \int_{x_{0}}^{X}\left[f_{1}(s, t) u^{q}\left(\tau_{1}(s), \tau_{2}(t)\right)+g_{1}(s, t) u^{r}\left(\tau_{1}(s), \tau_{2}(t)\right)\right] \Delta s \Delta t \\
& +\int_{y_{0}}^{y} \int_{x_{0}}^{X} \int_{y_{0}}^{t} \int_{x_{0}}^{s} h_{1}(\xi, \eta) u^{m}\left(\tau_{1}(\xi), \tau_{2}(\eta)\right) \Delta \xi \Delta \eta \Delta s \Delta t \\
\leq & a(X, y)+\mu+\int_{y_{0}}^{y} \int_{x_{0}}^{X}\left[f_{1}(s, t) v^{q / p}(s, t)+g_{1}(s, t) v^{r / p}(s, t)\right] \Delta s \Delta t \\
& +\int_{y_{0}}^{y} \int_{x_{0}}^{X} \int_{y_{0}}^{t} \int_{x_{0}}^{s} h_{1}(\xi, \eta) v^{m / p}(\xi, \eta) \Delta \xi \Delta \eta \Delta s \Delta t .
\end{aligned}
$$


Considering the structure of (2.45) is similar to (2.17), then following in a same manner as the process of (2.17)-(2.20) we can deduce

$$
\begin{aligned}
v(X, y) \leq & \mu+\widetilde{B}_{1}(X, y)+\int_{y_{0}}^{y} e_{\widetilde{B}_{2}}(y, \sigma(t)) \widetilde{B}_{2}(X, t)\left(\mu+\widetilde{B}_{1}(X, t)\right) \Delta t \\
= & \mu\left[1+\int_{y_{0}}^{y} e_{\widetilde{B}_{2}}(y, \sigma(t)) \widetilde{B}_{2}(X, t) \Delta t\right]+\widetilde{B}_{1}(X, y) \\
& +\int_{y_{0}}^{y} e_{\widetilde{B}_{2}}(y, \sigma(t)) \widetilde{B}_{2}(X, t) \widetilde{B}_{1}(X, t) \Delta t, \quad y \in\left[y_{0}, N\right] \bigcap \mathbb{T}
\end{aligned}
$$

where $\widetilde{B}_{1}(x, y), \widetilde{B}_{2}(x, y)$ are defined in (2.36) and (2.37), respectively.

Since $X$ is selected from $\left[x_{0}, M\right] \cap \mathbb{T}$ arbitrarily, then in fact (2.46) holds for all $x \in \mathbb{T}_{0}$, that is,

$$
\begin{aligned}
v(x, y) & \leq \mu\left[1+\int_{y_{0}}^{y} e_{\widetilde{B}_{2}}(y, \sigma(t)) \widetilde{B}_{2}(x, t) \Delta t\right]+\widetilde{B}_{1}(x, y)+\int_{y_{0}}^{y} e_{\widetilde{B}_{2}}(y, \sigma(t)) \widetilde{B}_{2}(x, t) \widetilde{B}_{1}(x, t) \Delta t \\
& =\mu \widetilde{B}_{3}(x, y)+\widetilde{B}_{4}(x, y), \quad(x, y) \in\left(\left[x_{0}, M\right] \bigcap \mathbb{T}\right) \times\left(\left[y_{0}, N\right] \bigcap \mathbb{T}\right)
\end{aligned}
$$

where $\widetilde{B}_{3}(x, y), \widetilde{B}_{4}(x, y)$ are defined in (2.38) and (2.39), respectively.

On the other hand, from (2.18), (2.42), and (2.44) we obtain

$$
\begin{aligned}
\mu \leq & \int_{y_{0}}^{N} \int_{x_{0}}^{M}\left[f_{2}(s, t) v^{q / p}(s, t)+g_{2}(s, t) v^{r / p}(s, t)+\int_{y_{0}}^{t} \int_{x_{0}}^{s} h_{2}(\xi, \eta) v^{m / p}(\xi, \eta) \Delta \xi \Delta \eta\right] \Delta s \Delta t \\
\leq & \int_{y_{0}}^{N} \int_{x_{0}}^{M}\left[f_{2}(s, t)\left(\frac{q}{p} K^{(q-p) / p} v(s, t)+\frac{p-q}{p} K^{q / p}\right)\right. \\
& \left.+g_{2}(s, t)\left(\frac{r}{p} K^{(r-p) / p} v(s, t)+\frac{p-r}{p} K^{r / p}\right)\right] \Delta s \Delta t \\
& +\int_{y_{0}}^{N} \int_{x_{0}}^{M} \int_{y_{0}}^{t} \int_{x_{0}}^{s} h_{2}(\xi, \eta)\left(\frac{m}{p} K^{(m-p) / p} v(\xi, \eta)+\frac{p-m}{p} K^{m / p}\right) \Delta \xi \Delta \eta \Delta s \Delta t \\
= & +\int_{y_{0}}^{N} \int_{x_{0}}^{M}\left[f_{2}(s, t) \frac{q}{p} K^{(q-p) / p} v(s, t)+g_{2}(s, t) \frac{r}{p} K^{(r-p) / p} v(s, t)\right] \Delta s \Delta t \\
& +\int_{y_{0}}^{N} \int_{x_{0}}^{M} \int_{y_{0}}^{t} \int_{x_{0}}^{s} h_{2}(\xi, \eta) \frac{m}{p} K^{(m-p) / p} v(\xi, \eta) \Delta \xi \Delta \eta \Delta s \Delta t
\end{aligned}
$$


where $\lambda$ is defined in (2.35). Then using (2.47) in (2.48) yields

$$
\begin{aligned}
\mu \leq & \lambda+\int_{y_{0}}^{N} \int_{x_{0}}^{M}\left\{f_{2}(s, t) \frac{q}{p} K^{(q-p) / p}\left[\mu \widetilde{B}_{3}(s, t)+\widetilde{B}_{4}(s, t)\right]\right. \\
& \left.+g_{2}(s, t) \frac{r}{p} K^{(r-p) / p}\left[\mu \widetilde{B}_{3}(s, t)+\widetilde{B}_{4}(s, t)\right]\right\} \Delta s \Delta t \\
& +\int_{y_{0}}^{N} \int_{x_{0}}^{M} \int_{y_{0}}^{t} \int_{x_{0}}^{s} h_{2}(\xi, \eta) \frac{m}{p} K^{(m-p) / p}\left[\mu \widetilde{B}_{3}(\xi, \eta)+\widetilde{B}_{4}(\xi, \eta)\right] \Delta \xi \Delta \eta \Delta s \Delta t \\
= & +\mu\left\{\int_{y_{0}}^{N} \int_{x_{0}}^{M}\left[f_{2}(s, t) \frac{q}{p} K^{(q-p) / p} \widetilde{B}_{3}(s, t)+g_{2}(s, t) \frac{r}{p} K^{(r-p) / p} \widetilde{B}_{3}(s, t)\right] \Delta s \Delta t\right. \\
& \left.+\int_{y_{0}}^{N} \int_{x_{0}}^{M} \int_{y_{0}}^{t} \int_{x_{0}}^{s} h_{2}(\xi, \eta) \frac{m}{p} K^{(m-p) / p} \widetilde{B}_{3}(\xi, \eta) \Delta \xi \Delta \eta \Delta s \Delta t\right\} \\
& +\int_{y_{0}}^{N} \int_{x_{0}}^{M}\left[f_{2}(s, t) \frac{q}{p} K^{(q-p) / p} \widetilde{B}_{4}(s, t)+g_{2}(s, t) \frac{r}{p} K^{(r-p) / p} \widetilde{B}_{4}(s, t)\right] \Delta s \Delta t \\
& +\int_{y_{0}}^{N} \int_{x_{0}}^{M} \int_{y_{0}}^{t} \int_{x_{0}}^{s} h_{2}(\xi, \eta) \frac{m}{p} K^{(m-p) / p} \widetilde{B}_{4}(\xi, \eta) \Delta \xi \Delta \eta \Delta s \Delta t \\
= & \lambda+\mu \widetilde{B}_{5}+\widetilde{B}_{6},
\end{aligned}
$$

which implies

$$
\mu \leq \frac{\lambda+\widetilde{B}_{6}}{1-\widetilde{B}_{5}}
$$

Combining (2.43), (2.47), and (2.50) we can obtain the desired inequality (2.34).

In the proof of Theorem 2.9, if we let the right side of (2.33) be $a(x, y)+v(x, y)$ in the first statement, then following in a same process as in Theorem 2.9 we obtain another bound on the function $u(x, y)$, which is shown in the following theorem.

Theorem 2.10. Under the conditions of Theorem 2.9, if, for $(x, y) \in\left(\left[x_{0}, M\right] \cap \mathbb{T}\right) \times\left(\left[y_{0}, N\right] \cap \mathbb{T}\right)$, $u(x, y)$ satisfies (2.33) with the initial condition (2.8), then the following inequality holds:

$$
u(x, y) \leq\left\{a(x, y)+\frac{\tilde{\mu}+J_{1}(x, y)}{1-\tilde{\jmath}} e_{J_{2}}\left(y, y_{0}\right)\right\}^{1 / p}, \quad(x, y) \in\left(\left[x_{0}, M\right] \bigcap \mathbb{T}\right) \times\left(\left[y_{0}, N\right] \bigcap \mathbb{T}\right)
$$


provided that $\tilde{\lambda}<1$, where

$$
\begin{aligned}
& \tilde{\mathcal{\lambda}}=\int_{y_{0}}^{N} \int_{x_{0}}^{M}\left[f_{2}(s, t) \frac{q}{p} K^{(q-p) / p} e_{J_{2}}\left(t, y_{0}\right)+g_{2}(s, t) \frac{r}{p} K^{(r-p) / p} e_{J_{2}}\left(t, y_{0}\right)\right] \Delta s \Delta t \\
& +\int_{y_{0}}^{N} \int_{x_{0}}^{M} \int_{y_{0}}^{t} \int_{x_{0}}^{s} h_{2}(\xi, \eta) \frac{m}{p} K^{(m-p) / p} e_{J_{2}}\left(\eta, y_{0}\right) \Delta \xi \Delta \eta \Delta s \Delta t, \\
& \tilde{\mu}=\int_{y_{0}}^{N} \int_{x_{0}}^{M}\left[f_{2}(s, t)\left(\frac{q}{p} K^{(q-p) / p} a(s, t)+\frac{p-q}{p} K^{q / p}\right)\right. \\
& +g_{2}(s, t)\left(\frac{r}{p} K^{(r-p) / p} a(s, t)+\frac{p-r}{p} K^{r / p}\right) \\
& +\int_{y_{0}}^{t} \int_{x_{0}}^{s} h_{2}(\xi, \eta)\left(\frac{m}{p} K^{(m-p) / p}(a(\xi, \eta)+v(\xi, \eta))\right. \\
& \left.\left.+\frac{p-m}{p} K^{m / p}\right) \Delta \xi \Delta \eta\right] \Delta s \Delta t \\
& J_{1}(x, y)=\int_{y_{0}}^{y} \int_{x_{0}}^{x}\left[f_{1}(s, t)\left(\frac{q}{p} K^{(q-p) / p} a(s, t)+\frac{p-q}{p} K^{q / p}\right)\right. \\
& \left.+g_{1}(s, t)\left(\frac{r}{p} K^{(r-p) / p} a(s, t)+\frac{p-r}{p} K^{r / p}\right)\right] \Delta s \Delta t \\
& +\int_{y_{0}}^{y} \int_{x_{0}}^{X} \int_{y_{0}}^{t} \int_{x_{0}}^{s} h_{1}(\xi, \eta)\left(\frac{m}{p} K^{(m-p) / p} a(\xi, \eta)+\frac{p-m}{p} K^{m / p}\right) \Delta \xi \Delta \eta \Delta s \Delta t, \\
& J_{2}(x, y)=\int_{x_{0}}^{x}\left[f_{1}(s, y) \frac{q}{p} K^{(q-p) / p}+g_{1}(s, y) \frac{r}{p} K^{(r-p) / p}+\int_{y_{0}}^{y} \int_{x_{0}}^{s} h_{1}(\xi, \eta) \frac{m}{p} K^{(m-p) / p} \Delta \xi \Delta \eta\right] \Delta s .
\end{aligned}
$$

Finally, we will establish a more general inequality than that in Theorems 2.9-2.10. Consider the following inequality:

$$
\begin{aligned}
u^{p}(x, y) \leq & a(x, y) \\
& +\int_{y_{0}}^{y} \int_{x_{0}}^{x}\left[L\left(s, t, u\left(\tau_{1}(s), \tau_{2}(t)\right)\right)+\int_{y_{0}}^{t} \int_{x_{0}}^{s} h_{1}(\xi, \eta) u^{q}\left(\tau_{1}(\xi), \tau_{2}(\eta)\right) \Delta \xi \Delta \eta\right] \Delta s \Delta t \\
& +\int_{y_{0}}^{N} \int_{x_{0}}^{M}\left[L\left(s, t, u\left(\tau_{1}(s), \tau_{2}(t)\right)\right)+\int_{y_{0}}^{t} \int_{x_{0}}^{s} h_{2}(\xi, \eta) u^{q}\left(\tau_{1}(\xi), \tau_{2}(\eta)\right) \Delta \xi \Delta \eta\right] \Delta s \Delta t
\end{aligned}
$$

with the initial condition (2.8), where $u, a, p, q, \phi, \alpha, \beta, \tau_{i}, h_{i}, i=1,2$ are the same as in Theorem 2.4, $M \in \mathbb{T}_{0}, N \in \widetilde{\mathbb{T}}_{0}$ are two fixed numbers. $L \in\left(\mathbb{T}_{0} \times \widetilde{\mathbb{T}}_{0} \times \mathbb{R}_{+}, \mathbb{R}_{+}\right)$, and $0 \leq L(s, t, x)-L(s, t, y) \leq A(s, t, y)(x-y)$ for $x \geq y \geq 0$, where $A \in\left(\mathbb{T}_{0} \times \widetilde{\mathbb{T}}_{0} \times \mathbb{R}_{+}, \mathbb{R}_{+}\right)$. 
Journal of Applied Mathematics

Theorem 2.11. If, for $(x, y) \in\left(\left[x_{0}, M\right] \cap \mathbb{T}\right) \times\left(\left[y_{0}, N\right] \cap \mathbb{T}\right), u(x, y)$ satisfies (2.53), then the following inequality holds:

$$
u(x, y) \leq\left\{\left[\frac{\widehat{\lambda}+\widehat{B}_{6}}{1-\widehat{B}_{5}}\right] \widehat{B}_{3}(x, y)+\widehat{B}_{4}(x, y)\right\}^{1 / p}, \quad(x, y) \in\left(\left[x_{0}, M\right] \bigcap \mathbb{T}\right) \times\left(\left[y_{0}, N\right] \bigcap \mathbb{T}\right)
$$

provided that $\widetilde{B}_{5}<1$, where

$$
\begin{aligned}
\hat{\mathcal{\lambda}}= & \int_{y_{0}}^{N} \int_{x_{0}}^{M}\left[L\left(s, t, \frac{p-1}{p} K^{1 / p}\right)+\int_{y_{0}}^{t} \int_{x_{0}}^{s} h_{2}(\xi, \eta) \frac{p-q}{p} K^{q / p} \Delta \xi \Delta \eta\right] \Delta s \Delta t, \\
\widehat{B}_{1}(x, y)= & a(x, y) \\
& +\int_{y_{0}}^{y} \int_{x_{0}}^{x}\left[L\left(s, t, \frac{p-1}{p} K^{1 / p}\right)+\int_{y_{0}}^{t} \int_{x_{0}}^{s} h_{1}(\xi, \eta) \frac{p-q}{p} K^{q / p} \Delta \xi \Delta \eta\right] \Delta s \Delta t, \quad \forall K>0, \\
\widehat{B}_{2}(x, y)= & \int_{x_{0}}^{x}\left[A\left(s, y, \frac{p-1}{p} K^{1 / p}\right) \frac{1}{p} K^{(1-p) / p}\right. \\
& \left.+\int_{y_{0}}^{y} \int_{x_{0}}^{s} h_{1}(\xi, \eta) \frac{q}{p} K^{(q-p) / p} \Delta \xi \Delta \eta\right] \Delta s, \quad \forall K>0, \\
\widehat{B}_{3}(x, y)= & 1+\int_{y_{0}}^{y} e_{\widehat{B}_{2}}(y, \sigma(t)) \widehat{B}_{2}(x, t) \Delta t \\
\widehat{B}_{4}(x, y)= & \widehat{B}_{1}(x, y)+\int_{y_{0}}^{y} e_{\widehat{B}_{2}}(y, \sigma(t)) \widehat{B}_{2}(x, t) \widehat{B}_{1}(x, t) \Delta t, \\
\widehat{B}_{5}= & \int_{y_{0}}^{N} \int_{x_{0}}^{M}\left[A\left(s, t, \frac{p-1}{p} K^{1 / p}\right) \frac{1}{p} K^{(1-p) / p} \widehat{B}_{3}(s, t)\right. \\
& \left.+\int_{y_{0}}^{t} \int_{x_{0}}^{s} h_{x_{0}}^{t}(\xi, \eta) \frac{q}{p} K^{(q-p) / p} \widehat{B}_{3}(\xi, \eta) \Delta \xi \Delta \eta\right] \Delta s \Delta t, \\
\widehat{B}_{6}= & \int_{y_{0}}^{N} \int_{x_{0}}^{M}\left[A\left(s, t, \frac{p-1}{p} K^{1 / p}\right) \frac{1}{p} K^{(1-p) / p} \widehat{B}_{4}(s, t)\right.
\end{aligned}
$$

Proof. Let the right side of $(2.53)$ be $v(x, y)$ and

$$
\widehat{\mu}=\int_{y_{0}}^{N} \int_{x_{0}}^{M}\left[L\left(s, t, u\left(\tau_{1}(s), \tau_{2}(t)\right)\right)+\int_{y_{0}}^{t} \int_{x_{0}}^{s} h_{2}(\xi, \eta) u^{q}\left(\tau_{1}(\xi), \tau_{2}(\eta)\right) \Delta \xi \Delta \eta\right] \Delta s \Delta t .
$$


Then

$$
u(x, y) \leq v^{1 / p}(x, y), \quad(x, y) \in\left(\left[x_{0}, M\right] \bigcap \mathbb{T}\right) \times\left(\left[y_{0}, N\right] \bigcap \mathbb{T}\right)
$$

Similar to the process of (2.14)-(2.16) we have

$$
u\left(\tau_{1}(x), \tau_{2}(y)\right) \leq v^{1 / p}(x, y), \quad(x, y) \in\left(\left[x_{0}, M\right] \bigcap \mathbb{T}\right) \times\left(\left[y_{0}, N\right] \bigcap \mathbb{T}\right)
$$

Fix $X \in\left[x_{0}, M\right] \cap \mathbb{T}$, and let $x \in\left[x_{0}, X\right] \cap \mathbb{T}, y \in\left[y_{0}, N\right] \cap \mathbb{T}$. Then

$$
\begin{aligned}
v(X, y)= & a(X, y)+\widehat{\mu} \\
& +\int_{y_{0}}^{y} \int_{x_{0}}^{X}\left[L\left(s, t, u\left(\tau_{1}(s), \tau_{2}(t)\right)\right)+\int_{y_{0}}^{t} \int_{x_{0}}^{s} h_{1}(\xi, \eta) u^{q}\left(\tau_{1}(\xi), \tau_{2}(\eta)\right) \Delta \xi \Delta \eta\right] \Delta s \Delta t \\
\leq & a(X, y)+\widehat{\mu}+\int_{y_{0}}^{y} \int_{x_{0}}^{X}\left[L\left(s, t, v^{1 / p}(s, t)\right)+\int_{y_{0}}^{t} \int_{x_{0}}^{s} h_{1}(\xi, \eta) v^{q / p}(\xi, \eta) \Delta \xi \Delta \eta\right] \Delta s \Delta t .
\end{aligned}
$$

From Lemma 2.3, we have

$$
\begin{gathered}
v^{q / p}(x, y) \leq \frac{q}{p} K^{(q-p) / p} v(x, y)+\frac{p-q}{p} K^{q / p}, \\
v^{1 / p}(x, y) \leq \frac{1}{p} K^{(1-p) / p} v(x, y)+\frac{p-1}{p} K^{1 / p}, \quad \forall K>0 .
\end{gathered}
$$

Combining (2.65) and (2.66), it follows that

$$
\begin{aligned}
v(X, y) \leq & a(X, y)+\widehat{\mu}+\int_{y_{0}}^{y} \int_{x_{0}}^{X} L\left(s, t, \frac{1}{p} K^{(1-p) / p} v(s, t)+\frac{p-1}{p} K^{1 / p}\right) \Delta s \Delta t \\
& +\int_{y_{0}}^{y} \int_{x_{0}}^{X} \int_{y_{0}}^{t} \int_{x_{0}}^{s} h_{1}(\xi, \eta)\left(\frac{q}{p} K^{(q-p) / p} v(\xi, \eta)+\frac{p-q}{p} K^{q / p}\right) \Delta \xi \Delta \eta \Delta s \Delta t \\
= & a(X, y)+\widehat{\mu} \\
& +\int_{y_{0}}^{y} \int_{x_{0}}^{X}\left[L\left(s, t, \frac{1}{p} K^{(1-p) / p} v(s, t)+\frac{p-1}{p} K^{1 / p}\right)-L\left(s, t, \frac{p-1}{p} K^{1 / p}\right)\right. \\
& \left.+L\left(s, t, \frac{p-1}{p} K^{1 / p}\right)\right] \Delta s \Delta t
\end{aligned}
$$


Journal of Applied Mathematics

$$
\begin{aligned}
&+\int_{y_{0}}^{y} \int_{x_{0}}^{X} \int_{y_{0}}^{t} \int_{x_{0}}^{s} h_{1}(\xi, \eta)\left(\frac{q}{p} K^{(q-p) / p} v(\xi, \eta)+\frac{p-q}{p} K^{q / p}\right) \Delta \xi \Delta \eta \Delta s \Delta t \\
& \leq aX, y)+\widehat{\mu} \\
&+\int_{y_{0}}^{y} \int_{x_{0}}^{X}\left[A\left(s, t, \frac{p-1}{p} K^{1 / p}\right) \frac{1}{p} K^{(1-p) / p} v(s, t)+L\left(s, t, \frac{p-1}{p} K^{1 / p}\right)\right] \Delta s \Delta t \\
&+\int_{y_{0}}^{y} \int_{x_{0}}^{X}\left[\int_{y_{0}}^{t} \int_{x_{0}}^{s} h_{1}(\xi, \eta) \frac{q}{p} K^{(q-p) / p} \Delta \xi \Delta \eta\right] v(X, t) \Delta s \Delta t \\
&+\int_{y_{0}}^{y} \int_{x_{0}}^{X} \int_{y_{0}}^{t} \int_{x_{0}}^{s} h_{1}(\xi, \eta) \frac{p-q}{p} K^{q / p} \Delta \xi \Delta \eta \Delta s \Delta t \\
& \leq a(X, y)+\widehat{\mu}+\int_{y_{0}}^{y}\left[\int_{x_{0}}^{X} A\left(s, t, \frac{p-1}{p} K^{1 / p}\right) \frac{1}{p} K^{(1-p) / p} \Delta s\right] v(X, t) \Delta t \\
&+\int_{y_{0}}^{y} \int_{x_{0}}^{X} L\left(s, t, \frac{p-1}{p} K^{1 / p}\right) \Delta s \Delta t \\
&+ \int_{y_{0}}^{y}\left[\int_{x_{0}}^{X} \int_{y_{0}}^{t} \int_{x_{0}}^{s} h_{1}(\xi, \eta) \frac{q}{p} K^{(q-p) / p} \Delta \xi \Delta \eta \Delta s\right] v(X, t) \Delta t \\
&+ \int_{y_{0}}^{y} \int_{x_{0}}^{X} \int_{y_{0}}^{t} \int_{x_{0}}^{s} h_{1}(X, y)+\int_{y_{0}}^{y} \widehat{B}_{2}(X, t) v(X, t) \Delta t, \\
& p
\end{aligned}
$$

where $\widehat{B}_{1}(x, y), \widehat{B}_{2}(x, y)$ are defined in (2.56) and (2.57), respectively.

We notice the structure of (2.67) is similar to (2.19), so following in a same manner as in (2.19)-(2.21) we obtain

$$
\begin{aligned}
& v(x, y) \\
& \quad \leq \widehat{\mu}\left[1+\int_{y_{0}}^{y} e_{\widehat{B}_{2}}(y, \sigma(t)) \widehat{B}_{2}(x, t) \Delta t\right]+\widehat{B}_{1}(x, y)+\int_{y_{0}}^{y} e_{\widehat{B}_{2}}(y, \sigma(t)) \widehat{B}_{2}(x, t) \widehat{B}_{1}(x, t) \Delta t \\
& \quad=\widehat{\mu} \widehat{B}_{3}(x, y)+\widehat{B}_{4}(x, y), \quad(x, y) \in\left(\left[x_{0}, M\right] \bigcap \mathbb{T}\right) \times\left(\left[y_{0}, N\right] \bigcap \mathbb{T}\right),
\end{aligned}
$$

where $\widehat{B}_{3}(x, y), \widehat{B}_{4}(x, y)$ are defined in (2.58) and (2.59), respectively. 
On the other hand, from (2.62), (2.64), and (2.66) we have

$$
\begin{aligned}
& \widehat{\mu} \leq \int_{y_{0}}^{N} \int_{x_{0}}^{M}\left[L\left(s, t, v^{1 / p}\right)+\int_{y_{0}}^{t} \int_{x_{0}}^{s} h_{2}(\xi, \eta) v^{q / p}(\xi, \eta) \Delta \xi \Delta \eta\right] \Delta s \Delta t \\
& \leq \int_{y_{0}}^{N} \int_{x_{0}}^{M}\left[L\left(s, t, \frac{1}{p} K^{(1-p) / p} v(s, t)+\frac{p-1}{p} K^{1 / p}\right)\right. \\
& \left.+\int_{y_{0}}^{t} \int_{x_{0}}^{s} h_{2}(\xi, \eta)\left(\frac{q}{p} K^{(q-p) / p} v(\xi, \eta)+\frac{p-q}{p} K^{q / p}\right) \Delta \xi \Delta \eta\right] \Delta s \Delta t \\
& =\int_{y_{0}}^{N} \int_{x_{0}}^{M}\left[L\left(s, t, \frac{1}{p} K^{(1-p) / p} v(s, t)+\frac{p-1}{p} K^{1 / p}\right)-L\left(s, t, \frac{p-1}{p} K^{1 / p}\right)\right. \\
& \left.+L\left(s, t, \frac{p-1}{p} K^{1 / p}\right)\right] \Delta s \Delta t \\
& +\int_{y_{0}}^{N} \int_{x_{0}}^{M} \int_{y_{0}}^{t} \int_{x_{0}}^{s} h_{2}(\xi, \eta)\left(\frac{q}{p} K^{(q-p) / p} v(\xi, \eta)+\frac{p-q}{p} K^{q / p}\right) \Delta \xi \Delta \eta \Delta s \Delta t \\
& \leq \int_{y_{0}}^{N} \int_{x_{0}}^{M}\left[A\left(s, t, \frac{p-1}{p} K^{1 / p}\right) \frac{1}{p} K^{(1-p) / p} v(s, t)+L\left(s, t, \frac{p-1}{p} K^{1 / p}\right)\right] \Delta s \Delta t \\
& +\int_{y_{0}}^{N} \int_{x_{0}}^{M} \int_{y_{0}}^{t} \int_{x_{0}}^{s} h_{2}(\xi, \eta)\left(\frac{q}{p} K^{(q-p) / p} v(\xi, \eta)+\frac{p-q}{p} K^{q / p}\right) \Delta \xi \Delta \eta \Delta s \Delta t \\
& =\widehat{\jmath}+\int_{y_{0}}^{N} \int_{x_{0}}^{M}\left[A\left(s, t, \frac{p-1}{p} K^{1 / p}\right) \frac{1}{p} K^{(1-p) / p} v(s, t)\right. \\
& \left.+\int_{y_{0}}^{t} \int_{x_{0}}^{s} h_{2}(\xi, \eta) \frac{q}{p} K^{(q-p) / p} v(\xi, \eta) \Delta \xi \Delta \eta\right] \Delta s \Delta t
\end{aligned}
$$

where $\hat{\lambda}$ is defined in (2.55). Then using (2.68) in (2.69) yields

$$
\begin{aligned}
& \widehat{\mu} \leq \widehat{\imath}+\int_{y_{0}}^{N} \int_{x_{0}}^{M} A\left(s, t, \frac{p-1}{p} K^{1 / p}\right) \frac{1}{p} K^{(1-p) / p}\left[\widehat{\mu} \widehat{B}_{3}(s, t)+\widehat{B}_{4}(s, t)\right] \Delta s \Delta t \\
& +\int_{y_{0}}^{N} \int_{x_{0}}^{M} \int_{y_{0}}^{t} \int_{x_{0}}^{s} h_{2}(\xi, \eta) \frac{q}{p} K^{(q-p) / p}\left[\widehat{\mu} \widehat{B}_{3}(\xi, \eta)+\widehat{B}_{4}(\xi, \eta)\right] \Delta \xi \Delta \eta \Delta s \Delta t \\
& =\widehat{\lambda}+\widehat{\mu}\left\{\int_{y_{0}}^{N} \int_{x_{0}}^{M} A\left(s, t, \frac{p-1}{p} K^{1 / p}\right) \frac{1}{p} K^{(1-p) / p} \widehat{B}_{3}(s, t)\right. \\
& \left.+\int_{y_{0}}^{t} \int_{x_{0}}^{s} h_{2}(\xi, \eta) \frac{q}{p} K^{(q-p) / p} \widehat{B}_{3}(\xi, \eta) \Delta \xi \Delta \eta \Delta s \Delta t\right\}
\end{aligned}
$$




$$
\begin{aligned}
& +\int_{y_{0}}^{N} \int_{x_{0}}^{M}\left[A\left(s, t, \frac{p-1}{p} K^{1 / p}\right) \frac{1}{p} K^{(1-p) / p} \widehat{B}_{4}(s, t)\right. \\
& \left.\quad+\int_{y_{0}}^{t} \int_{x_{0}}^{s} h_{2}(\xi, \eta) \frac{q}{p} K^{(q-p) / p} \widehat{B}_{4}(\xi, \eta) \Delta \xi \Delta \eta\right] \Delta s \Delta t \\
& =\hat{\jmath}+\widehat{\mu} \widehat{B}_{5}+\widehat{B}_{6},
\end{aligned}
$$

which implies

$$
\widehat{\mu} \leq \frac{\widehat{\imath}+\widehat{B}_{6}}{1-\widehat{B}_{5}} .
$$

Combining (2.63), (2.68), and (2.71), we obtain the desired inequality (2.54).

Remark 2.12. The established above inequalities generalize many known results including both integral inequalities for continuous functions and discrete inequalities. For example, if we take $\mathbb{T}=\mathbb{R}, p=q=1, g(x, y)=h(x, y) \equiv 0$, then Theorem 2.4 reduces to [1, Theorem 2.2], which is one case of integral inequality for continuous function. If we take $\mathbb{T}=\mathbb{R}, h(x, y) \equiv$ $0, a(x, y) \equiv C$, then Corollary 2.7 reduces to [2, Theorem 3 (c1)], which is another case of inequality for continuous function. If we take $\mathbb{T}=\mathbb{R}, p=q=1, g_{1}(x, y)=g_{2}(x, y)=f_{2}(x, y)=$ $h_{1}(x, y)=h_{2}(x, y) \equiv 0$, then Theorem 2.10 reduces to [1, Theorem 2.2] with slight difference. If we take $\mathbb{T}=\mathbb{Z}, g_{1}(x, y)=h_{1}(x, y)=f_{2}(x, y)=h_{2}(x, y) \equiv 0, \tau_{1}(x)=x, \tau_{2}(y)=y$, then Theorem 2.10 reduces to [3, Theorem 2.1], which is a discrete inequality.

Remark 2.13. Since $\mathbb{T}$ is an arbitrary time scale, then if we take $\mathbb{T}$ for some peculiar cases, such as $\mathbb{T}=\mathbb{R}$ or $\mathbb{T}=\mathbb{Z}$, we can deduce a series of corollaries according to Theorem 2.4-2.11. Due to the limited space, we omit them here.

\section{Some Applications}

In this section, we will present some applications for the results we have established previously. New explicit bounds for solutions of certain dynamic equations are derived in the first two examples, while the quantitative property of solutions is focused on in the final example.

Example 3.1. Consider the following delay dynamic differential equation:

$$
\begin{aligned}
& \left(u^{p}(x, y)\right)_{y x}^{\Delta \Delta} \\
& =F\left(s, t, u\left(\tau_{1}(s), \tau_{2}(t)\right), \int_{y_{0}}^{t} \int_{x_{0}}^{s} W\left(\xi, \eta, u\left(\tau_{1}(\xi), \tau_{2}(\eta)\right)\right) \Delta \xi \Delta \eta\right), \quad(x, y) \in \mathbb{T}_{0} \times \widetilde{\mathbb{T}}_{0},
\end{aligned}
$$


with the initial condition

$$
\begin{gathered}
\left(u^{p}\left(x_{0}, y\right)\right)_{y}^{\Delta}=b^{\Delta}(y), \quad u^{p}\left(x, y_{0}\right)=a(x), \\
u(x, y)=\phi(x, y), \quad \text { if } x \in\left[\alpha, x_{0}\right] \bigcap \mathbb{T}, \text { or } y \in\left[\beta, y_{0}\right] \bigcap \mathbb{T}, \\
\left|\phi\left(\tau_{1}(x), \tau_{2}(y)\right)\right| \leq|k(x, y)|^{1 / p}, \quad \text { if } \tau_{1}(x) \leq x_{0}, \text { or } \tau_{2}(y) \leq y_{0}, \forall(x, y) \in \mathbb{T}_{0} \times \widetilde{\mathbb{T}}_{0},
\end{gathered}
$$

where $u \in C_{\mathrm{rd}}\left(\mathbb{T}_{0} \times \widetilde{\mathbb{T}}_{0}, \mathbb{R}\right), a \in C_{\mathrm{rd}}\left(\mathbb{T}_{0}, \mathbb{R}\right), b \in C_{\mathrm{rd}}\left(\widetilde{\mathbb{T}}_{0}, \mathbb{R}\right), b$ is delta differential, and $b\left(y_{0}\right)=$ $0, k \in C_{\mathrm{rd}}\left(\mathbb{T}_{0} \times \widetilde{\mathbb{T}}_{0}, \mathbb{R}_{+}\right), p>0$ is a constant, $\phi \in C_{\mathrm{rd}}\left(\left(\left[\alpha, x_{0}\right] \times\left[\beta, y_{0}\right]\right) \cap \mathbb{T}^{2}, \mathbb{R}\right), F \in\left(\mathbb{T}_{0} \times \widetilde{\mathbb{T}}_{0} \times\right.$ $\left.\mathbb{R}^{2}, \mathbb{R}\right), W \in\left(\mathbb{T}_{0} \times \widetilde{\mathbb{T}}_{0} \times \mathbb{R}, \mathbb{R}\right) . \alpha, \beta, \tau_{1}, \tau_{2}$ are the same as in Theorem 2.4 .

Theorem 3.2. Suppose that $u(x, y)$ is a solution of (3.1)-(3.2), $|a(x)+b(y)| \leq k(x, y)$, and $|F(s, t, x, y)| \leq f(s, t)|x|^{q}+|y|,|W(\xi, \eta, x)| \leq h(\xi, \eta)|x|^{m}$, where $f, h, q, m$ are defined as in Theorem 2.4; then

$$
|u(x, y)| \leq\left[B_{1}(x, y)+\int_{y_{0}}^{y} e_{B_{2}}(y, \sigma(t)) B_{2}(x, t) B_{1}(x, t) \Delta t\right]^{1 / p}, \quad(x, y) \in \mathbb{T}_{0} \times \widetilde{\mathbb{T}}_{0}
$$

where

$$
\begin{array}{r}
B_{1}(x, y)=k(x, y)+\int_{y_{0}}^{y} \int_{x_{0}}^{x}\left[f(s, t) \frac{p-q}{p} K^{q / p}+\int_{y_{0}}^{t} \int_{x_{0}}^{s} h(\xi, \eta) \frac{p-m}{p} K^{m / p} \Delta \xi \Delta \eta\right] \Delta s \Delta t, \\
\forall K>0,
\end{array}
$$

and $B_{2}(x, y)$ is defined as in Theorem 2.4 (with $g(x, y) \equiv 0$ ).

Proof. The equivalent integral equation of (3.1) can be denoted by

$$
\begin{aligned}
u^{p}(x, y)= & a(x)+b(y) \\
& +\int_{y_{0}}^{y} \int_{x_{0}}^{x} F\left(s, t, u\left(\tau_{1}(s), \tau_{2}(t)\right), \int_{y_{0}}^{t} \int_{x_{0}}^{s} W\left(\xi, \eta, u\left(\tau_{1}(\xi), \tau_{2}(\eta)\right)\right) \Delta \xi \Delta \eta\right) \Delta s \Delta t .
\end{aligned}
$$

Then

$$
\begin{aligned}
& \left|u^{p}(x, y)\right| \\
& \quad \leq k(x, y)+\int_{y_{0}}^{y} \int_{x_{0}}^{x}\left|F\left(s, t, u\left(\tau_{1}(s), \tau_{2}(t)\right), \int_{y_{0}}^{t} \int_{x_{0}}^{s} W\left(\xi, \eta, u\left(\tau_{1}(\xi), \tau_{2}(\eta)\right)\right) \Delta \xi \Delta \eta\right)\right| \Delta s \Delta t
\end{aligned}
$$


Journal of Applied Mathematics

$$
\begin{aligned}
& \leq k(x, y) \\
& +\int_{y_{0}}^{y} \int_{x_{0}}^{x}\left[f(s, t)\left|u\left(\tau_{1}(s), \tau_{2}(t)\right)\right|^{q}+\left|\int_{y_{0}}^{t} \int_{x_{0}}^{s} W\left(\xi, \eta, u\left(\tau_{1}(\xi), \tau_{2}(\eta)\right)\right) \Delta \xi \Delta \eta\right|\right] \Delta s \Delta t \\
& \leq k(x, y)+\int_{y_{0}}^{y} \int_{x_{0}}^{x}\left[f(s, t)\left|u\left(\tau_{1}(s), \tau_{2}(t)\right)\right|^{q}\right. \\
& \left.+\int_{y_{0}}^{t} \int_{x_{0}}^{s} h(\xi, \eta)\left|u\left(\tau_{1}(\xi), \tau_{2}(\eta)\right)\right|^{m} \Delta \xi \Delta \eta\right] \Delta s \Delta t
\end{aligned}
$$

and a suitable application of Theorem 2.4 to (3.6) yields the desired inequality (3.3).

Theorem 3.3. Under the conditions of Theorem 3.2, one has

$$
|u(x, y)| \leq\left[B_{1}(x, y) e_{B_{2}}\left(y, y_{0}\right)\right]^{1 / p}, \quad(x, y) \in \mathbb{T}_{0} \times \widetilde{\mathbb{T}}_{0},
$$

where $B_{1}, B_{2}$ are defined as in Theorem 3.2.

Proof. The desired inequality can be obtained by an application of Theorem 2.5 to (3.6).

Example 3.4. Consider the following delay dynamic integral equation:

$$
\begin{array}{r}
u^{p}(x, y)=C+\int_{y_{0}}^{y} \int_{x_{0}}^{x} F_{1}\left(s, t, u\left(\tau_{1}(s), \tau_{2}(t)\right), \int_{y_{0}}^{t} \int_{x_{0}}^{s} W_{1}\left(\xi, \eta, u\left(\tau_{1}(\xi), \tau_{2}(\eta)\right)\right) \Delta \xi \Delta \eta\right) \Delta s \Delta t \\
+\int_{y_{0}}^{N} \int_{x_{0}}^{M} F_{2}\left(s, t, u\left(\tau_{1}(s), \tau_{2}(t)\right), \int_{y_{0}}^{t} \int_{x_{0}}^{s} W_{2}\left(\xi, \eta, u\left(\tau_{1}(\xi), \tau_{2}(\eta)\right)\right) \Delta \xi \Delta \eta\right) \Delta s \Delta t, \\
(x, y) \in\left(\left[x_{0}, M\right] \bigcap \mathbb{T}\right) \times\left(\left[y_{0}, N\right] \bigcap \mathbb{T}\right),
\end{array}
$$

with the initial condition

$$
\begin{gathered}
u(x, y)=\phi(x, y), \quad \text { if } x \in\left[\alpha, x_{0}\right] \bigcap \mathbb{T} \text {, or } y \in\left[\beta, y_{0}\right] \bigcap \mathbb{T}, \\
\left|\phi\left(\tau_{1}(x), \tau_{2}(y)\right)\right| \leq|C|^{1 / p}, \quad \text { if } \tau_{1}(x) \leq x_{0}, \text { or } \tau_{2}(y) \leq y_{0}, \forall(x, y) \in \mathbb{T}_{0} \times \widetilde{\mathbb{T}}_{0},
\end{gathered}
$$

where $u \in C_{\mathrm{rd}}\left(\mathbb{T}_{0} \times \widetilde{\mathbb{T}}_{0}, \mathbb{R}\right), p>0$ is a constant, $C=u^{p}\left(x_{0}, y_{0}\right), M \in \mathbb{T}_{0}, N \in \widetilde{\mathbb{T}}_{0}$ are two fixed numbers. $\phi \in C_{\text {rd }}\left(\left(\left[\alpha, x_{0}\right] \times\left[\beta, y_{0}\right]\right) \cap \mathbb{T}^{2}, \mathbb{R}\right), F_{i} \in\left(\mathbb{T}_{0} \times \widetilde{\mathbb{T}}_{0} \times \mathbb{R}^{2}, \mathbb{R}\right), \quad W_{i} \in\left(\mathbb{T}_{0} \times \widetilde{\mathbb{T}}_{0} \times \mathbb{R}, \mathbb{R}\right), i=$ 1,2. $\alpha, \beta, \tau_{1}, \tau_{2}$ are the same as in Theorem 2.4. 
Theorem 3.5. Suppose $u(x, y)$ is a solution of (3.8)-(3.9) and $\left|F_{i}(s, t, x, y)\right| \leq L(s, t,|x|)+$ $|y|,\left|W_{i}(\xi, \eta, x)\right| \leq h_{i}(\xi, \eta)|x|^{q}, i=1,2$, where $L, h_{i}, i=1,2, q$ are defined the same as in Theorem 2.11; then the following inequality holds:

$$
|u(x, y)| \leq\left\{\left[\frac{\widehat{\lambda}+\widehat{B}_{6}}{1-\widehat{B}_{5}}\right] \widehat{B}_{3}(x, y)+\widehat{B}_{4}(x, y)\right\}^{1 / p}, \quad(x, y) \in\left(\left[x_{0}, M\right] \bigcap \mathbb{T}\right) \times\left(\left[y_{0}, N\right] \bigcap \mathbb{T}\right)
$$

provided that $\widehat{B}_{5}<1$, where $\widehat{\lambda}, \widehat{B}_{2}(x, y), \widehat{B}_{3}(x, y), \widehat{B}_{4}(x, y), \widehat{B}_{5}, \widehat{B}_{6}$ are defined the same as in Theorem 2.11, and

$$
\begin{array}{r}
\widehat{B}_{1}(x, y)=|C|+\int_{y_{0}}^{y} \int_{x_{0}}^{x}\left[L\left(s, t, \frac{p-1}{p} K^{1 / p}\right)+\int_{y_{0}}^{t} \int_{x_{0}}^{s} h_{1}(\xi, \eta) \frac{p-q}{p} K^{q / p} \Delta \xi \Delta \eta\right] \Delta s \Delta t \\
\forall K>0 .
\end{array}
$$

Proof. From (3.8) we have

$$
\begin{aligned}
& \left|u^{p}(x, y)\right| \\
& \leq|C| \\
& +\int_{y_{0}}^{y} \int_{x_{0}}^{x}\left|F_{1}\left(s, t, u\left(\tau_{1}(s), \tau_{2}(t)\right), \int_{y_{0}}^{t} \int_{x_{0}}^{s} W_{1}\left(\xi, \eta, u\left(\tau_{1}(\xi), \tau_{2}(\eta)\right)\right) \Delta \xi \Delta \eta\right)\right| \Delta s \Delta t \\
& +\int_{y_{0}}^{N} \int_{x_{0}}^{M}\left|F_{2}\left(s, t, u\left(\tau_{1}(s), \tau_{2}(t)\right), \int_{y_{0}}^{t} \int_{x_{0}}^{s} W_{2}\left(\xi, \eta, u\left(\tau_{1}(\xi), \tau_{2}(\eta)\right)\right) \Delta \xi \Delta \eta\right)\right| \Delta s \Delta t \\
& \leq|C| \\
& +\int_{y_{0}}^{y} \int_{x_{0}}^{x}\left[L\left(s, t,\left|u\left(\tau_{1}(s), \tau_{2}(t)\right)\right|\right)+\left|\int_{y_{0}}^{t} \int_{x_{0}}^{s} W_{1}\left(\xi, \eta, u\left(\tau_{1}(\xi), \tau_{2}(\eta)\right)\right) \Delta \xi \Delta \eta\right|\right] \Delta s \Delta t \\
& +\int_{y_{0}}^{N} \int_{x_{0}}^{M}\left[L\left(s, t,\left|u\left(\tau_{1}(s), \tau_{2}(t)\right)\right|\right)+\left|\int_{y_{0}}^{t} \int_{x_{0}}^{s} W_{2}\left(\xi, \eta, u\left(\tau_{1}(\xi), \tau_{2}(\eta)\right)\right) \Delta \xi \Delta \eta\right|\right] \Delta s \Delta t \\
& \leq|C| \\
& +\int_{y_{0}}^{y} \int_{x_{0}}^{x}\left[L\left(s, t,\left|u\left(\tau_{1}(s), \tau_{2}(t)\right)\right|\right)+\int_{y_{0}}^{t} \int_{x_{0}}^{s} h_{1}(\xi, \eta)\left|u\left(\tau_{1}(\xi), \tau_{2}(\eta)\right)\right|^{q} \Delta \xi \Delta \eta\right] \Delta s \Delta t \\
& +\int_{y_{0}}^{N} \int_{x_{0}}^{M}\left[L\left(s, t,\left|u\left(\tau_{1}(s), \tau_{2}(t)\right)\right|\right)+\int_{y_{0}}^{t} \int_{x_{0}}^{s} h_{2}(\xi, \eta)\left|u\left(\tau_{1}(\xi), \tau_{2}(\eta)\right)\right|^{q} \Delta \xi \Delta \eta\right] \Delta s \Delta t .
\end{aligned}
$$


So by use of Theorem 2.11 we obtain the desired inequality (3.10).

Example 3.6. Consider the following delay dynamic integral equation:

$$
\begin{aligned}
& u(x, y) \\
& =C+\int_{y_{0}}^{y} \int_{x_{0}}^{x} F\left(s, t, u\left(\tau_{1}(s), \tau_{2}(t)\right), \int_{y_{0}}^{t} \int_{x_{0}}^{s} W\left(\xi, \eta, u\left(\tau_{1}(\xi), \tau_{2}(\eta)\right)\right) \Delta \xi \Delta \eta\right) \Delta s \Delta t,
\end{aligned}
$$

where $u \in C_{\mathrm{rd}}\left(\mathbb{T}_{0} \times \widetilde{\mathbb{T}}_{0}, \mathbb{R}\right), C=u^{p}\left(x_{0}, y_{0}\right), F \in\left(\mathbb{T}_{0} \times \widetilde{\mathbb{T}}_{0} \times \mathbb{R}^{2}, \mathbb{R}\right), W \in\left(\mathbb{T}_{0} \times \widetilde{\mathbb{T}}_{0} \times \mathbb{R}, \mathbb{R}\right) . \tau_{1}, \tau_{2}$ are the same as in Theorem 2.4.

Theorem 3.7. Assume that $\left|F\left(s, t, u_{1}, v_{1}\right)-F\left(s, t, u_{2}, v_{2}\right)\right| \leq f(s, t)\left|u_{1}-u_{2}\right|+\left|v_{1}-v_{2}\right|, \mid W\left(s, t, u_{1}\right)-$ $W\left(s, t, u_{2}\right)|\leq h(s, t)| u_{1}-u_{2} \mid$, where $f$, $h$ are defined as in Theorem 2.4, and; furthermore, assume that $\tau_{1}(x) \geq x_{0}, \tau_{2}(y) \geq y_{0}$, then (3.13) has at most one solution.

Proof. Suppose that $u_{1}(x, y), u_{2}(x, y)$ are two solutions of (3.13); then we have

$$
\begin{aligned}
& \left|u_{1}(x, y)-u_{2}(x, y)\right| \\
& \leq \mid \int_{y_{0}}^{y} \int_{x_{0}}^{x}\left[F\left(s, t, u_{1}\left(\tau_{1}(s), \tau_{2}(t)\right) \int_{y_{0}}^{t} \int_{x_{0}}^{s} W\left(\xi, \eta, u_{1}\left(\tau_{1}(\xi), \tau_{2}(\eta)\right)\right) \Delta \xi \Delta \eta\right)\right. \\
& \left.-F\left(s, t, u_{2}\left(\tau_{1}(s), \tau_{2}(t)\right) \int_{y_{0}}^{t} \int_{x_{0}}^{s} W\left(\xi, \eta, u_{2}\left(\tau_{1}(\xi), \tau_{2}(\eta)\right)\right) \Delta \xi \Delta \eta\right)\right] \Delta s \Delta t \\
& \leq \int_{y_{0}}^{y} \int_{x_{0}}^{x} \mid F\left(s, t, u_{1}\left(\tau_{1}(s), \tau_{2}(t)\right) \int_{y_{0}}^{t} \int_{x_{0}}^{s} W\left(\xi, \eta, u_{1}\left(\tau_{1}(\xi), \tau_{2}(\eta)\right)\right) \Delta \xi \Delta \eta\right) \\
& -F\left(s, t, u_{2}\left(\tau_{1}(s), \tau_{2}(t)\right) \int_{y_{0}}^{t} \int_{x_{0}}^{s} W\left(\xi, \eta, u_{2}\left(\tau_{1}(\xi), \tau_{2}(\eta)\right)\right) \Delta \xi \Delta \eta\right) \mid \Delta s \Delta t \\
& \leq \int_{y_{0}}^{y} \int_{x_{0}}^{x} f(s, t)\left|u_{1}\left(\tau_{1}(s), \tau_{2}(t)\right)-u_{2}\left(\tau_{1}(s), \tau_{2}(t)\right)\right| \Delta s \Delta t \\
& +\int_{y_{0}}^{y} \int_{x_{0}}^{x} \int_{y_{0}}^{t} \int_{x_{0}}^{s}\left|W\left(\xi, \eta, u_{1}\left(\tau_{1}(\xi), \tau_{2}(\eta)\right)\right)-W\left(\xi, \eta, u_{2}\left(\tau_{1}(\xi), \tau_{2}(\eta)\right)\right)\right| \Delta \xi \Delta \eta \Delta s \Delta t
\end{aligned}
$$




$$
\begin{aligned}
& \leq \int_{y_{0}}^{y} \int_{x_{0}}^{x} f(s, t)\left|u_{1}\left(\tau_{1}(s), \tau_{2}(t)\right)-u_{2}\left(\tau_{1}(s), \tau_{2}(t)\right)\right| \Delta s \Delta t \\
& \quad+\int_{y_{0}}^{y} \int_{x_{0}}^{x} \int_{y_{0}}^{t} \int_{x_{0}}^{s} h(\xi, \eta)\left|u_{1}\left(\tau_{1}(\xi), \tau_{2}(\eta)\right)-u_{2}\left(\tau_{1}(\xi), \tau_{2}(\eta)\right)\right| \Delta \xi \Delta \eta \Delta s \Delta t .
\end{aligned}
$$

Then a suitable application of Theorem 2.8 yields $\left|u_{1}(x, y)-u_{2}(x, y)\right| \leq 0$, that is, $u_{1}(x, y) \equiv$ $u_{2}(x, y)$, and the proof is complete.

\section{Conclusions}

In this paper, we established some new Gronwall-Bellman type integral inequalities on time scales. As one can see, the presented results provide a handy tool in the quantitative as well as qualitative analysis of solutions of certain delay dynamic equations on time scales. The established inequalities unify some known continuous and discrete inequalities.

\section{Acknowledgments}

This work is supported by Natural Science Foundation of Shandong Province (ZR2009AM011) China and Specialized Research Fund for the Doctoral Program of Higher Education (20103705110003) China. The authors thank the referees very much for their careful comments and valuable suggestions on this paper.

\section{References}

[1] H. X. Zhang and F. W. Meng, "Integral inequalities in two independent variables for retarded Volterra equations," Applied Mathematics and Computation, vol. 199, no. 1, pp. 90-98, 2008.

[2] B. G. Pachpatte, "Explicit bounds on certain integral inequalities," Journal of Mathematical Analysis and Applications, vol. 267, no. 1, pp. 48-61, 2002.

[3] Q.H. Ma, "Estimates on some power nonlinear Volterra-Fredholm type discrete inequalities and their applications," Journal of Computational and Applied Mathematics, vol. 233, no. 9, pp. 2170-2180, 2010.

[4] L. Ou-Iang, "The boundedness of solutions of linear differential equations $y^{\prime \prime}+A(t) y=0$," Shuxue Jinzhan, vol. 3, pp. 409-418, 1957.

[5] B. G. Pachpatte, "On some new inequalities related to a certain inequality arising in the theory of differential equations," Journal of Mathematical Analysis and Applications, vol. 251, no. 2, pp. 736-751, 2000.

[6] S. F. Deng, "Nonlinear discrete inequalities with two variables and their applications," Applied Mathematics and Computation, vol. 217, no. 5, pp. 2217-2225, 2010.

[7] O. Lipovan, "Integral inequalities for retarded Volterra equations," Journal of Mathematical Analysis and Applications, vol. 322, no. 1, pp. 349-358, 2006.

[8] Q. H. Ma and J. Pečarić, "Some new nonlinear retarded integral inequalities and their applications," Mathematical Inequalities and Applications, vol. 9, no. 4, pp. 617-632, 2006.

[9] P. Y. H. Pang and R. P. Agarwal, "On an integral inequality and its discrete analogue," Journal of Mathematical Analysis and Applications, vol. 194, no. 2, pp. 569-577, 1995.

[10] B. G. Pachpatte, "On some fundamental integral inequalities and their discrete analogues," Journal of Inequalities in Pure and Applied Mathematics, vol. 2, article 10, no. 2, 2001.

[11] R. P. Agarwal, S. F. Deng, and W. N. Zhang, "Generalization of a retarded Gronwall-like inequality and its applications," Applied Mathematics and Computation, vol. 165, no. 3, pp. 599-612, 2005. 
[12] R. A. C. Ferreira and D. F. M. Torres, "Generalized retarded integral inequalities," Applied Mathematics Letters, vol. 22, no. 6, pp. 876-881, 2009.

[13] W. S. Cheung and Q. H. Ma, "On certain new Gronwall-Ou-Iang type integral inequalities in two variables and their applications," Journal of Inequalities and Applications, vol. 8, pp. 347-361, 2005.

[14] S. Hilger, "Analysis on measure chains-a unified approach to continuous and discrete calculus," Results in Mathematics, vol. 18, pp. 18-56, 1990.

[15] M. Bohner, L. Erbe, and A. Peterson, "Oscillation for nonlinear second order dynamic equations on a time scale," Journal of Mathematical Analysis and Applications, vol. 301, no. 2, pp. 491-507, 2005.

[16] M. J. Bohner, R. A. C. Ferreira, and D. F. M. Torres, "Integral inequalities and their applications to the calculus of variations on time scales," Mathematical Inequalities and Applications, vol. 13, no. 3, pp. 511-522, 2010.

[17] R. P. Agarwal, M. Bohner, D. O'Regan, and A. Peterson, "Dynamic equations on time scales: a survey," Journal of Computational and Applied Mathematics, vol. 141, no. 1-2, pp. 1-26, 2002.

[18] F. H. Wong, C. C. Yeh, and W. C. Lian, "An extension of Jensen's inequality on time scales," Advances in Dynamical Systems and Applications, vol. 1, no. 1, pp. 113-120, 2006.

[19] H. M. Srivastava, K. L. Tseng, S. J. Tseng, and J. C. Lo, "Some generalizations of maronis inequality on time scales," Mathematical Inequalities E Applications, vol. 14, no. 2, pp. 469-480, 2011.

[20] M. Bohner and T. Matthews, "The Grüss inequality on time scales," Commun. Math. Anal., vol. 3, no. 1, pp. 1-8, 2007.

[21] Q. A. Ngô, "Some mean value theorems for integrals on time scales," Applied Mathematics and Computation, vol. 213, no. 2, pp. 322-328, 2009.

[22] W. J. Liu and Q. A. Ngô, "Some Iyengar-type inequalities on time scales for functions whose second derivatives are bounded," Applied Mathematics and Computation, vol. 216, pp. 3244-3251, 2010.

[23] W. N. Li, "Some new dynamic inequalities on time scales," Journal of Mathematical Analysis and Applications, vol. 319, no. 2, pp. 802-814, 2006.

[24] R. Agarwal, M. Bohner, and A. Peterson, "Inequalities on time scales: a survey," Mathematical Inequalities and Applications, vol. 4, no. 4, pp. 535-557, 2001.

[25] W. N. Li, "Some Pachpatte type inequalities on time scales," Computers and Mathematics with Applications, vol. 57, no. 2, pp. 275-282, 2009.

[26] A. Tuna and S. Kutukcu, "Some integral inequalities on time scales," Applied Mathematics and Mechanics, vol. 29, no. 1, pp. 23-29, 2008.

[27] W. N. Li, "Some delay integral inequalities on time scales," Computers and Mathematics with Applications, vol. 59, no. 6, pp. 1929-1936, 2010.

[28] Q. H. Ma and J. Pečarić, "The bounds on the solutions of certain two-dimensional delay dynamic systems on time scales," Computers and Mathematics with Applications, vol. 61, pp. 2158-2163, 2011.

[29] M. Bohner and A. Peterson, Dynamic Equations on Time Scales: An Introduction with Applications, Birkhäuser, Boston, Mass, USA, 2001.

[30] F. C. Jiang and F. W. Meng, "Explicit bounds on some new nonlinear integral inequalities with delay," Journal of Computational and Applied Mathematics, vol. 205, no. 1, pp. 479-486, 2007. 


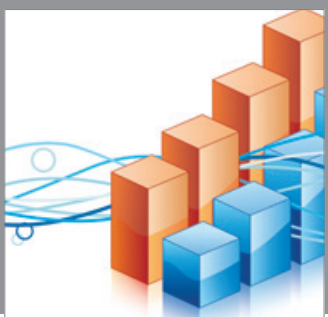

Advances in

Operations Research

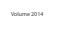

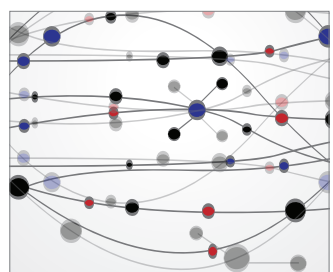

\section{The Scientific} World Journal
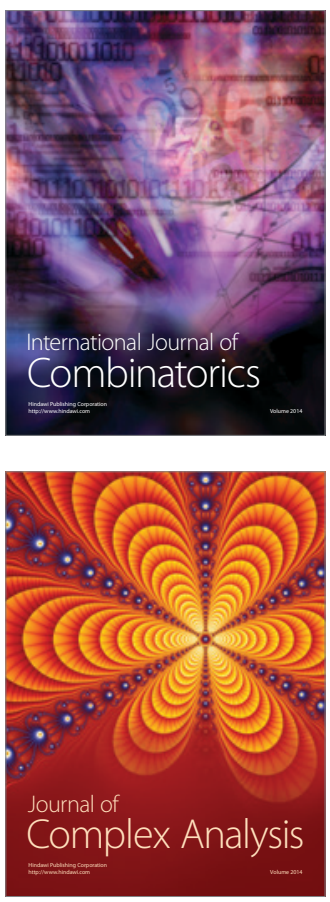

International Journal of

Mathematics and

Mathematical

Sciences
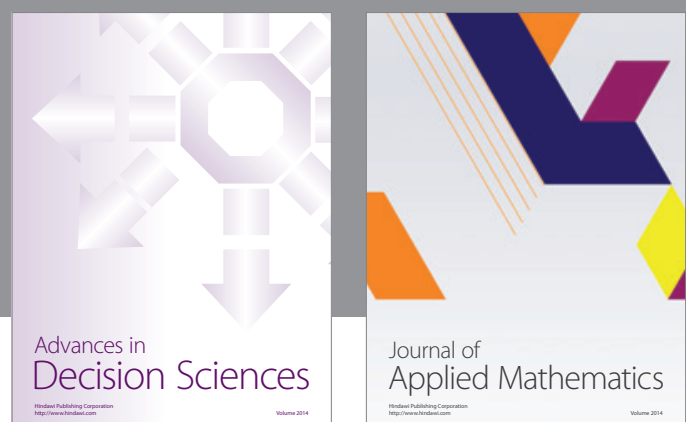

Journal of

Applied Mathematics
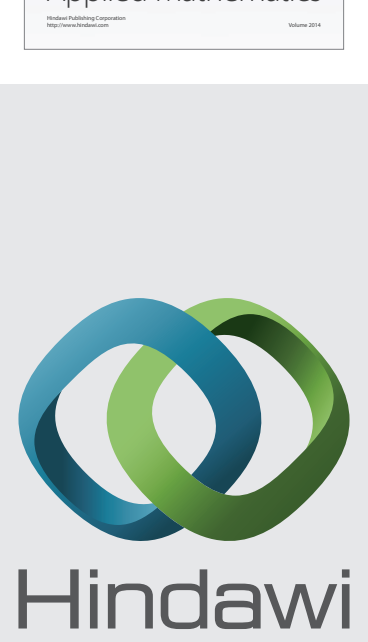

Submit your manuscripts at http://www.hindawi.com
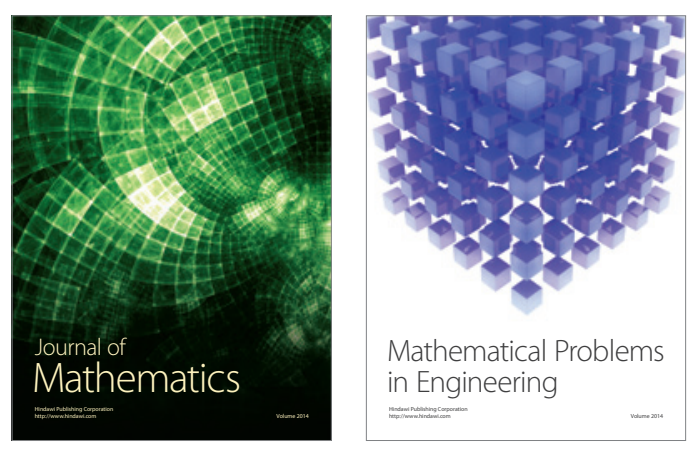

Mathematical Problems in Engineering
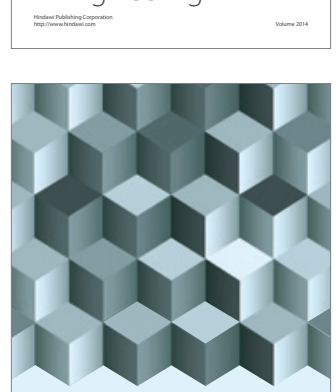

Journal of

Function Spaces
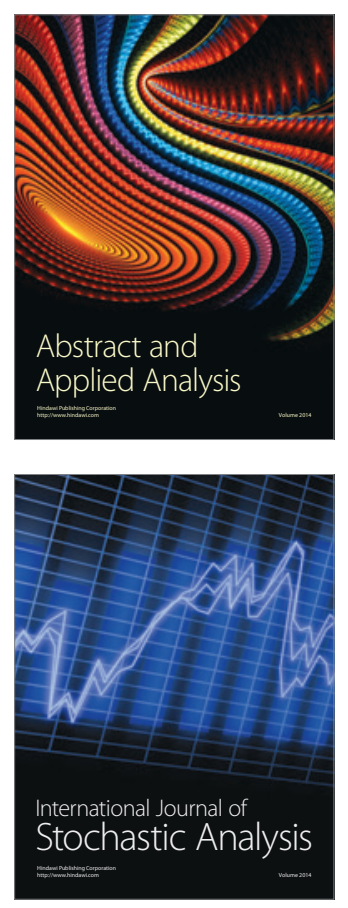

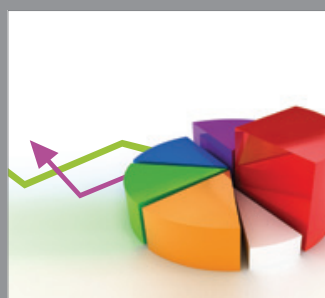

ournal of

Probability and Statistics

Promensencen
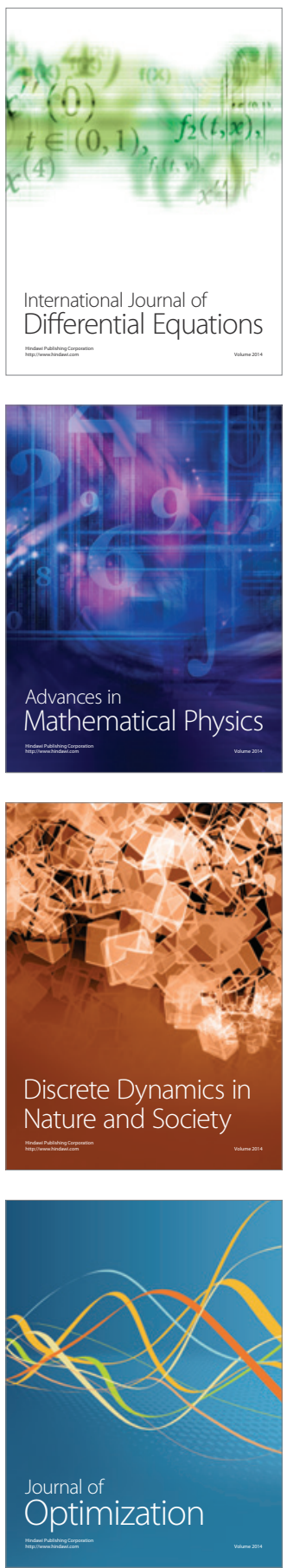